\title{
A multi-scale three-dimensional Cellular Automata fracture model of radiolytically oxidised nuclear graphite ${ }^{1}$
}

\author{
Yelena Vertyagina", Thomas James Marrow \\ University of Oxford, Parks Road, Oxford OX1 3PH, UK
}

\begin{abstract}
A multi-scale approach for fracture simulation, based on the Cellular Automata technique, has been developed and then applied to a nuclear graphite that is used in structural components of the UK Advanced Gas-cooled Reactors (AGR). High resolution X-ray computed tomographs of Gilsocarbon grade graphite, with up to $68 \%$ weight loss by radiolytic oxidation, provide quantitative descriptions of the porosity within its constitutive filler particles and their surrounding matrix. The statistical distributions for tensile strength and elastic modulus obtained from small models of the filler and matrix are introduced to a large scale model of the heterogeneous microstructure. These microstructure-derived simulations achieve a good agreement with experimental data. The computationally efficient analysis is then used to investigate the stochastic effects on mechanical properties of possible variations in the microstructure between individual small test specimens. As these may represent material of nominally the same microstructure, there is an apparent increase in the variability of strength and modulus at high weight loss.
\end{abstract}

Nomenclature of symbols and abbreviations

\begin{tabular}{|c|l|}
\hline AGR & Advanced Gas-cooled Reactor \\
\hline CA & Cellular Automata \\
\hline FE & Finite elements \\
\hline XFEM & Extended finite element method \\
\hline CAFE & Cellular Automata and Finite element technique \\
\hline HPB & Hinkley Point B \\
\hline EDN & Equivalent DIDO neutron \\
\hline DYM & Dynamic Young's modulus \\
\hline SCF & Stress concentration factor \\
\hline SED & Strain energy density \\
\hline SE & Strain energy \\
\hline$P$ & Porosity \\
\hline$T P$ & Total porosity \\
\hline$F P, M P$ & Average filler and matrix porosities correspondingly \\
\hline
\end{tabular}

\footnotetext{
${ }^{1}$ This is the author's copy of a paper accepted for publication in the journal 'Carbon' in 2017

* Corresponding author. E-mail: yelena.vertyagina@materials.ox.ac.uk (Yelena Veryagina)
} 


\begin{tabular}{|c|l|}
\hline$F, M, L$ & Filler, matrix and large pores fractions correspondingly \\
\hline$W$ & Weight loss \\
\hline$\rho$ & Density \\
\hline$\rho_{c}$ & Density of the graphite crystal \\
\hline$\sigma_{1}, \sigma_{2}, \sigma_{3}$ & Principal stresses \\
\hline$\sigma_{r r}, \sigma_{r \vartheta}, \sigma_{\phi \vartheta}, \sigma_{\phi \phi}$ & Stress components \\
\hline$p$ & Uniaxial tensile stress \\
\hline$R$ & Void radius \\
\hline$r$ & Distance from the void centre \\
\hline$v$ & Poisson's ratio \\
\hline$N$ & Number of active cells \\
\hline$a$ & Packing geometry factor \\
\hline$n$ & Pore geometry factor \\
\hline$E_{0}$ & Young's modulus for a pore-free material \\
\hline$E$ & Young's modulus for the porous material \\
\hline$A_{\text {surf }}$ & $\begin{array}{l}\text { The free surface area of all the matrix cells contacting the pores } \\
\text { orthogonally to the direction of tension }\end{array}$ \\
\hline$A_{\text {total }}$ & $\begin{array}{l}\text { The total free surface area of all the 'solid' cells surrounding the } \\
\text { pore }\end{array}$ \\
\hline$k$ & Topological factor \\
\hline$\omega_{i}$ & Local state of the cell with number $i$ \\
\hline$\Omega$ & The global state of the model \\
\hline$\sigma_{i j} \varepsilon_{i} U_{i}$ & Local values of the stress, strain and strain energy \\
\hline$\sigma_{a p p,} \varepsilon_{a p p}$ & Applied stress and strain \\
\hline$U_{\text {total }}$ & The global value of elastic energy in the system \\
\hline &
\end{tabular}

\section{Introduction}

Nuclear graphite has a complex and porous microstructure, and serves as a neutron moderator in nuclear reactors [1] for which knowledge of its structural properties is important to assure their safe operation. The AGR Gilsocarbon grade nuclear graphite has a porosity of around $20 \%$ in its initial "virgin" state, but as a result of irradiation and radiolytic oxidation that occur over the lifetime of these $\mathrm{CO}_{2}$-cooled reactors, the graphite's microstructure and properties change $[2,3]$. Neutron irradiation affects directly the graphite crystallites, and the changes in their properties can be described by pinning and structure factors that depend on the fast neutron dose [4]. Radiolytic oxidation affects the pore structure and progressively increases the total porosity, with a secondary effect on the evolution of the neutron dosedependent structure factor [5]. Nuclear graphite has porosity over a wide length scale from the fine accommodation cracks $[6,7]$ to larger gas porosity, and radiolytic oxidation causes the most significant changes in the smaller pores (area $\left.<100 \mu \mathrm{m}^{2}\right)[8]$.

The porosity of a material affects its mechanical properties; for instance, increasing pore content decreases fracture strength, the elastic and shear moduli and the thermal resistance, as 
shown by example in the literature that include sintered alumina $[9,10]$, cement and concrete $[10,11]$ and magnesium oxide [12]. These dependencies have a close relation to the volume fraction of porosity $[9,11,12]$, and are also sensitive to the pore geometry (size, shape, and orientation) [10]. Pores are concentrators of stress, and crack nucleation and growth in porous brittle materials is affected by the resultant non-uniform stress distribution within the microstructure.

This paper is concerned with the effects of radiolytic oxidation on the key mechanical properties of elastic modulus and strength in AGR graphite. The aim is to develop a model that gives improved confidence in the physical understanding of the quite significant effects that porosity evolution has on these properties. Graphites for nuclear applications are typically manufactured from calcined cokes, re-impregnated with coal or petroleum pitches, so that after graphitization at temperatures in excess of $2500{ }^{\circ} \mathrm{C}$ the resulting heterogeneous microstructure comprises filler particles within a matrix containing ground filler "flour" and graphitized pitch. The overall material contains pores and flaws arising from manufacture [13]. Crack nucleation in Gilsocarbon nuclear graphite, studied under different stress states $[14,15]$, has been shown to initiate from the larger pores, or agglomerations of large pores. Predictive models need to simulate the effects of the pore population on the stochastic property of strength [16], which is affected by the microstructure, and such models need to consider the real geometry of the complex pores that exist in nuclear graphite. The effects of component geometry are also important; for instance, a combined experimental-theoretical approach for studying the size effect on the fracture resistance of graphite was proposed in [17], proposing a modified maximum tangential stress fracture criterion that determined the extent of fracture process zone around the crack tip. As the graphite pore size ranges from several nanometres to several millimetres [18], a multi-scale modelling approach is required.

Empirical models have been developed that describe the evolution of strength [19] and elastic modulus [20] for Gilsocarbon graphite, accounting for both irradiation and oxidation effects. In the un-irradiated virgin condition, polygranular nuclear graphite generally has non-linear elastic properties, but these become linear with irradiation [21]. These models do not include a detailed consideration of graphite's microstructure and are fits to experimental data obtained over a limited range of oxidation, which limits their extrapolation to higher levels of oxidation. Such models also are not able to examine the sensitivity of mechanical properties to microstructure variations; for instance, the ratio of filler to matrix that may differ between 
small test specimens, and the spatial distribution of the larger pores that may be affected by processing and choices in raw materials in the manufacture of graphites.

Microstructure-sensitive simulations of nuclear graphite mechanical behaviour have been performed, for instance with the use of extended finite element methods (XFEM) [22]. However, the pore shape, size and distribution were not taken into account and the filler content was constant for all the considered microstructures. Three-dimensional (3D) finite element method (FEM) models that directly represent the pores in polygranular graphite microstructures during thermal [23] and radiolytical oxidation [24] have been created on the basis of computed X-ray tomography images. In that approach, both the visibly observable pores and the effects of non-observable porosity were considered, the latter quantified via the measured X-ray attenuation and hence the local material density. The obtained exponential decay of the bulk strength and elastic modulus with increasing porosity corresponded well with the available experimental data for both radiolytic and thermal oxidation of the material, although the models were both mesh and scale sensitive. The mechanical properties of nuclear graphite have also been addressed by multi-scale models [25], developed with FEM at the millimetre and centimetre length scales to simulate the effects of increasing porosity. However, in this case the pore structure was simplified as spheres, and no effect of pore geometry could be considered. Recent developments using multi-scale experimental characterisation and modelling have demonstrated a dominant effect of large pores on graphite strength and elastic modulus [26], and the collective behaviour of micro-cracks in graphite was considered using a 3D site-bond model for tensile strength [27]. This approach was subsequently developed into a dual scale model [28] for virgin Gilsocarbon graphite. However, the effect of large pores on the fracture process was not considered, nor was the influence of oxidation.

Despite significant advances in FE methods and parallel computing [29], the stochastic assessment of microstructure effects on physical properties is still a challenge. For an effective evaluation and prediction of the evolution of the physical properties of porous materials, a computationally efficient and microstructure-based model is still necessary. Here we propose and develop a novel and efficient Cellular Automata (CA) solution for the general problem of stress concentration and fracture in porous materials. The CA simulation takes into account the pore geometry and the direction of the applied load, and uses defined local mechanical characteristics of the matrix between pores, such as Young's modulus and Poisson's ratio. We then simulate the mechanical properties of radiolytically oxidised 
graphite over a significant range of weight loss, using a morphological analysis of the observed porosity as input for a multi-scale model.

\section{Morphological analysis of radiolytically oxidised graphite}

Laboratory X-ray computed tomography datasets of radiolytically oxidised Gilsocarbon graphite microstructures were provided by EDF Energy Generation, and a morphological analysis of their porous structure was conducted. All were obtained from material specified for the same AGR station (Hinkley Point B, HPB), and were thus manufactured by AGL (Anglo Great Lakes) similarly in terms of raw materials and processing; the specimens form part of an accelerated aging test programme [30]. Ten datasets with nominal weight loss by oxidation from $8.1 \%$ to $68.2 \%$ were selected for characterisation; the weight loss, also provided by EDF Energy Generation, is calculated from physical density measurements and is relative to the average density of virgin graphite of the same grade. The tomography data comprised pairs of 32-bit greyscale scans for each of 10 samples, obtained with an image voxel size of $2.5 \mu \mathrm{m}$. Each image was converted to 8-bit data and processed with a binary segmentation procedure using Avizo image analysis software (Avizo Fire 9.0) after a median filter was applied to the original image stack to reduce image noise. The pore volume was marked by the "magic wand" tool, selecting the large interconnected pores and the "tophat" tool using adaptive kernel sizes to select the closed pores in the microstructure.

Microstructures of the examined samples, alongside example extracted sub-volumes of a filler particle and matrix, are shown in Figure 1 for the lowest weight loss of $8.1 \%$, for an intermediate state with $41.5 \%$ weight loss, and for the highest weight loss of $68.2 \%$. The dimensions of each scan (approximately $5 \times 5 \times 3.5 \mathrm{~mm}$ ) and the weight loss values are reported in Table 1. The average observable porosity ranged from $17 \%$ to $73 \%$ with increasing weight loss. A fraction of the porosity is not available for segmentation due to the limited resolution of the tomographs; the virgin porosity, $P$, is $20.1 \%$ for this graphite according to Eq. (1) with virgin density $\rho=1.805 \mathrm{~g} / \mathrm{cm}^{3}[31]$ and the theoretical density of the graphite crystal $\rho_{c}=2.26 \mathrm{~g} / \mathrm{cm}^{3}$.

$$
P=\frac{\rho_{c}-\rho}{\rho_{c}}
$$

The identifiable filler particle fraction was measured in one tomography scan for each weight loss (Figure 2a) and decreases exponentially with the weight loss value. Filler particles can be recognised by their characteristic lenticular porosity, which has been used previously to 
identify such regions in microstructures of graphite by their fractal dimensions. By backextrapolation of the fitting function, the virgin graphite would have a filler fraction of large particles of $\sim 62 \%$. It was not possible to verify experimentally this extrapolation for virgin graphite; although filler particle regions can be identified in high resolution tomographs [32], the matrix porosity was not well resolved in the virgin HPB graphite and so it was not possible to reliably quantify the filler fraction.

Table 1. Characteristics of the radiolytically oxidised Hinkley Point B (HPB) graphite samples: dimensions of tomography scans, average weight loss (from density measurement), observable porosity, total fast neutron dose (equivalent DIDO neutron, EDN) and measured dynamic Young's modulus (DYM). The tomographs and data for weight loss, dose and DYM were provided by EDF Energy Generation.

\begin{tabular}{|c|c|c|c|c|c|}
\hline & $\begin{array}{l}\text { Tomography volume dimensions, mm } \\
\text { (Scan 1/ Scan 2) }\end{array}$ & $\begin{array}{c}\text { Nominal } \\
\text { weight loss, } \\
\%\end{array}$ & $\begin{array}{c}\text { Observable total } \\
\text { porosity, \% } \\
\text { (Scan } 1 \text { / Scan } 2)\end{array}$ & $\begin{array}{l}\text { Total dose, } \\
10^{20} \mathrm{n} / \mathrm{cm}^{2} \\
\text { EDN }\end{array}$ & $\begin{array}{c}\text { Measured } \\
\text { DYM, } \\
\text { GPa }\end{array}$ \\
\hline 1 & $4.88 \times 4.88 \times 3.27 / 4.86 \times 4.87 \times 3.16$ & 8.1 & $17.0 / 19.6$ & 95.3 & 20.8 \\
\hline 2 & $4.95 \times 4.95 \times 3.56 / 4.92 \times 4.92 \times 3.76$ & 11.2 & $21.2 / 21.3$ & 92.2 & 17.6 \\
\hline 3 & $4.79 \times 4.79 \times 3.89 / 4.78 \times 4.78 \times 4.45$ & 12.2 & $21.8 / 23.2$ & 114.5 & 18.4 \\
\hline 4 & $4.58 \times 4.58 \times 3.32 / 4.48 \times 4.47 \times 3.65$ & 14.2 & $25.8 / 23.9$ & 102.8 & 19.5 \\
\hline 5 & $4.89 \times 4.87 \times 3.31 / 4.90 \times 4.89 \times 3.78$ & 27.5 & $37.2 / 37.1$ & 95.0 & 11.5 \\
\hline 6 & $4.75 \times 4.82 \times 3.84 / 4.75 \times 4.81 \times 3.78$ & 34.7 & $41.4 / 46.3$ & 106.3 & 9.1 \\
\hline 7 & $4.90 \times 4.90 \times 3.24 / 4.90 \times 4.90 \times 4.35$ & 41.5 & $43.5 / 50.3$ & 154.8 & 6.6 \\
\hline 8 & $4.75 \times 4.75 \times 4.07 / 4.90 \times 4.90 \times 4.10$ & 53.0 & $58.5 / 57.5$ & 173.6 & 2.9 \\
\hline 9 & $4.94 \times 4.94 \times 3.65 / 4.92 \times 4.92 \times 3.61$ & 61.2 & $65.5 / 66.1$ & 183.4 & - \\
\hline 10 & $4.91 \times 4.91 \times 3.38 / 4.89 \times 4.89 \times 1.92$ & 68.2 & $71.7 / 72.9$ & 181.8 & - \\
\hline
\end{tabular}




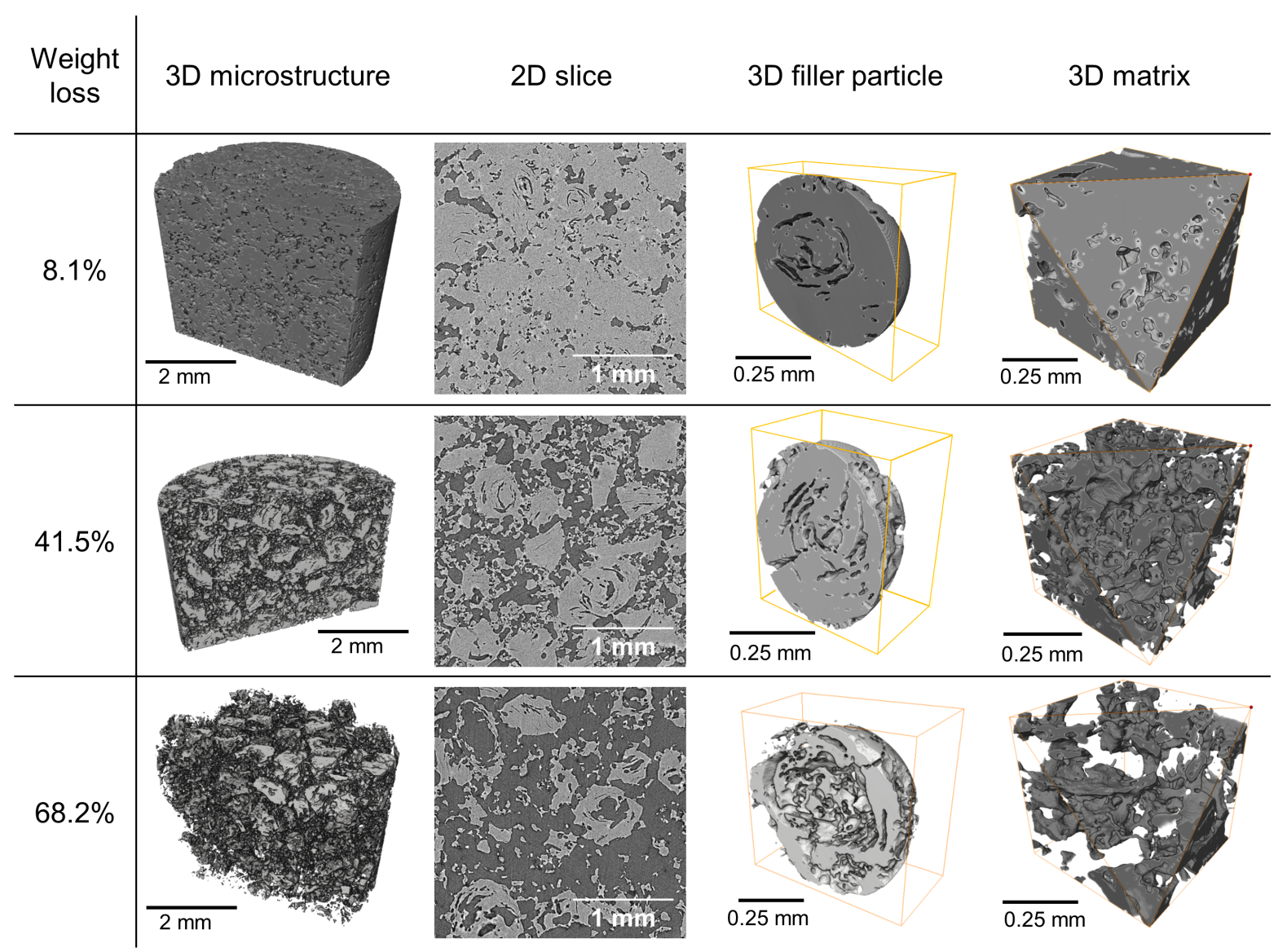

Figure 1 - Microstructure of radiolytically oxidised HPB graphite with different weight losses: 3D microstructure, 2D slice of the microstructure, 3D microstructures of a filler particle and a matrix region.

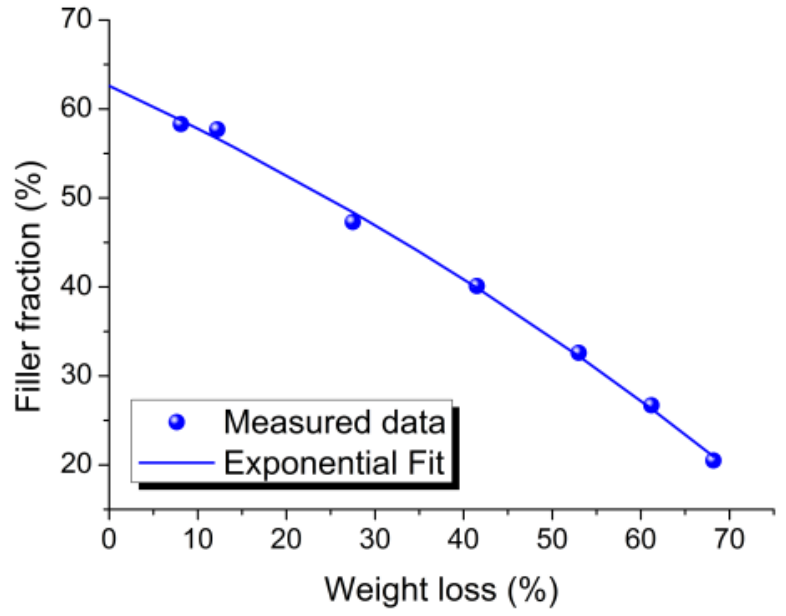

a)



b) 


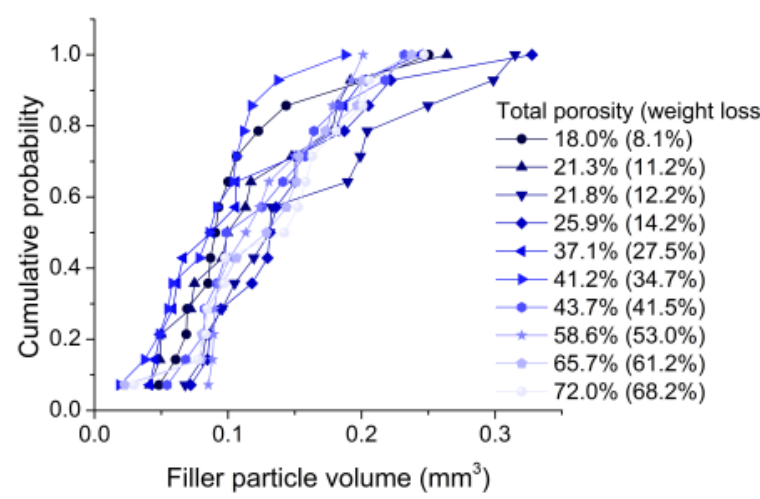

c)



d)

Figure 2 - Data measured with tomographs of radiolytically oxidised HPB graphite: a) observed filler fraction as a function of weight loss; b) mean porosities for filler and matrix samples obtained at each weight loss (error bars are the standard deviation of the measurements); c) filler particle volume distributions; d) filler particle size aspect ratio distributions. The total observed porosity (and the reported weight loss) of the bulk sample are shown in the legend.

To characterise the matrix porosity, 7 samples of matrix volumes with a size of $0.5 \mathrm{~mm}^{3}$ were extracted randomly from each of the irradiated graphite datasets; this was the largest volume that could be extracted reliably between the filler particles. The pore shapes are quite different in the matrix from those in the filler, and with increasing oxidation the matrix pores become spatially combined into a single pore, which occupies more than $90 \%$ of the extracted volume. To characterise the internal porosity and dimensions of the filler particles, 14 filler particles were extracted randomly from each of the datasets. The sizes of the filler particles are shown in Figure 2c; all the volumes are in the interval of $0.02-0.3 \mathrm{~mm}^{3}$ and have comparable aspect ratios (Figure 2d), calculated from the maximum and minimum dimensions. The mean porosity evolution in the extracted filler particles and matrix regions is shown in Figure $2 b$. It is notable that the matrix porosity is significantly higher than the filler porosity at high weight loss. These data, which describe the oxidised Gilsocarbon graphite microstructure, can be used in a multi-scale fracture model that takes into account the filler/matrix phase composition, the fine porosity (including pore geometry) within the filler particles and matrix sub-volumes, and also the larger scale porosity. The development of this model is described in the next section.

\section{Multiscale concept for microstructure based fracture modelling in porous graphite}

The simplest multiscale approach should include at least two scales. A small scale model must directly refer to a microstructure of a material under study and reflect its basic features such as pore geometry and the porosity effect on mechanical properties of the material 
including stress, elastic and shear moduli. This model may provide a statistical distribution of heterogeneous properties within the microstructure. A large scale model must account for these statistical data obtained from the small scale model and operate with the sample geometry at different length scales.

\subsection{D modelling for crack initiation and propagation in quasi-brittle porous materials}

Many models have been proposed to introduce the influence of pore geometry into simulations of the properties of materials, although there are few studies that have directly coupled the measured pore characteristics to a model. For instance, the authors in [33] proposed a function describing each pore shape and orientation; and in [34] a generalised cellular method was used to simulate the effects of regular pore shapes on Young's modulus, Poisson's ratio and the inelastic response (i.e. yield strength) of a well characterised glass that contained spherical pores, achieving good agreement with measured elastic properties (e.g. of Coble [9]). Both of these works consider only one type of regular porosity within the microstructure. More recently, in simulations using analytical and FEM for the effects of thermal [23, 35] and radiolytic [24] oxidation of nuclear graphite, the morphological characteristics of the larger pores were predicted to have a dominant stress concentrating effect. The characteristics of the pore population need to be considered explicitly, therefore, to achieve a better description in predictive models of the relationships between the bulk properties of a material and its microstructure.

The problem of the stress distribution in inhomogeneous materials has been solved exactly for elastic materials with the use of particular mathematical functions, but only for spherical pores and in single type porosity problems. This was demonstrated in [36] with the use of the minimum solid area concept, in [37] using phase-field modelling, and in [38] on the basis of a zero-order model. The problem of the stress distribution in complex porous microstructures can be solved only by approximate numerical methods. The geometrical effect of a small three-dimensional clusters of pores (up to 3 in number) on the stress concentration factor was investigated in [39] for spot-welds in steel, but most of the works in the literature that consider significant numbers of pores only describe 2D models, which have lower computational costs than 3D models. This imposes certain restrictions on the use of the results, as there is always the open question of whether a $2 \mathrm{D}$ model is sufficiently representative to reflect the properties of a $3 \mathrm{D}$ microstructure. 
To study fracture, a 3D model is needed that can simulate the patterns of stress and hence the strain energy for a complex distribution of pores. Such a model should also be capable ultimately of accommodating non-linear properties (i.e. a damage law) to adequately reflect the links between the microstructure and its physical properties. One novel approach to this problem, applied to concrete, used X-ray computed tomography observations of the microstructure to develop 3D lattice model simulations that achieved quite realistic predictions of the crack path [40]. Other methods that apply discrete elements include the quasi-static approach used in [41] in a study of porous ceramics' elastic behaviour on the basis of 3D aggregates of overlapping spheres, whilst the load redistribution with damage in ordered systems (exemplified by fibre composites) has been approximated using Green's functions [42].

In the context of nuclear graphite, small scale models may represent each of the two microstructure phases: either filler particles or matrix, taking into account their internal porosity as a third phase with null-properties. Using individual sub-volumes of the filler particles and matrix (i.e. as identified in Figure 2), one can develop small scale models for their individual mechanical behaviour. This would provide a statistical distribution of the failure stress values and local Young's modulus from their corresponding pore size distributions. Then, on the basis of experimental data for the filler and large pore size distributions, a large scale model can be created, where each component associated with the microstructure has mechanical properties that correspond to the chosen constituent, thus providing heterogeneity of material properties. We propose a Cellular Automata-based model for realisation of this problem.

\subsection{Description of the Cellular Automata (CA) solution for the stress field of a pore}

Cellular automata solutions represent a discrete system of a limited number of cells [43], which form a regular CA lattice. The lattice is used to represent the microstructure, so certain features of the microstructure are approximated by the CA cells. The cells may have different states and follow rules for evolution, such as mechanical damage. The states of these cells change synchronously at every simulation step and the cell states are determined by the current environment of each cell. CA provide a mathematical alternative to differential equations, so advantages arise because CA can operate in problems where differential equations cannot, such as discrete systems and complex shape boundaries. CA methods can provide flexible rules for evolution of the system where local interactions in the model lead to the global behaviour of the entire system; for example [44]. Any boundary conditions can be 
applied to the model, including periodic boundaries to exclude undesired edge effects in a finite volume.

Numerical 3D models on the basis of $\mathrm{CA}$ for the simulation of fracture processes at the microstructural scale have been suggested previously. For example, in [45] the elasto-plastic CA approach was used to simulate crack initiation and damage development in rocks under uniaxial compression; however, although the material was heterogeneous in the mechanical parameters that were applied to each cell according to a given distribution, pores were not considered. A computer simulation tool based on the excitable CA method was proposed in [46] for calculating the local moments and accumulated elastic energy. A 3D damage model, using the movable CA technique, is considered in [47] following the original approach proposed in [48]; the interactions between the cells are programmed as particle interactions, and pores are represented explicitly as structural elements of the CA-lattice. This allows their effect on the stress distribution in the material to be considered. However, the computational complexity of the particle method increases greatly with the number of the elements, and this restricts the size and resolution of the model. A 3D CA-finite element (CAFE)-based model has also been developed in [49], which may be used to predict damage development in quasibrittle materials with porous microstructures [50]. However, that model is currently restricted to simple pore shapes.

The main disadvantage of CA analysis arises from its discrete nature, which can affect the reproducibility of certain physical phenomena where a global state is described by a physical equation developed for continuum medium. CA often operates with unit-less parameters, so calibration and validation are therefore very important. The rules for the CA operating in the model described in this paper are based on the equations of the theory of elasticity and are validated against analytical solutions. The stress tensor for uniaxial tension of an infinite 3D linear elastic solid with a central spherical void [51] can be written in the spherical coordinate system [52] as in equation (1):

$$
\left[\begin{array}{ccc}
\sigma_{r r} & \sigma_{r \theta} & 0 \\
\sigma_{r \theta} & \sigma_{\theta \theta} & 0 \\
0 & 0 & \sigma_{\varphi \varphi}
\end{array}\right]
$$

where the stress components are calculated as [52]:

$$
\sigma_{r r}=p \cos ^{2} \theta+p \frac{\rho^{3}}{7-5 v}\left(6\left(1-\rho^{2}\right)-\left(5(5-v)-18 \rho^{2}\right) \cos ^{2} \theta\right)
$$




$$
\begin{gathered}
\sigma_{\theta \theta}=p \sin ^{2} \theta+p \frac{\rho^{3}}{7-5 v}\left(4-5 v+9 \rho^{2}+\left(5(1-2 v)-21 \rho^{2}\right) \cos ^{2} \theta\right) \\
\sigma_{\varphi \varphi}=p \frac{3 \rho^{3}}{7-5 v}\left(-2+5 v+\rho^{2}+5\left(1-2 v-\rho^{2}\right) \cos ^{2} \theta\right) \\
\sigma_{r \theta}=-p \sin ^{2} 2 \theta\left(\frac{1}{2}+\frac{\rho^{3}}{2(7-5 v)}\left(5(1+v)-12 \rho^{2}\right)\right) \\
\rho=\frac{R}{r}
\end{gathered}
$$

where $p$ is the uniaxial tensile stress in the $z$-direction, $R$ is the void radius, $r$ is the distance from the void centre, and $v$ is Poisson's ratio. Three principal stresses $\sigma_{1}, \sigma_{2}, \sigma_{3}$ can be found through the stress invariants.

CA can be programmed so that different cells represent the behaviour of the various elements of the inhomogeneous structure; each cell type operates individually. Thus a solid containing a void can be regarded as a set of cellular automata with two main states:

- The first state corresponds to the basic state of the solid without inhomogeneous features. These inactive elements of the structure are the cells outside of the void.

- The second state is responsible for the geometry of the void. The cells inside the void have this state and are considered as active elements of the CA lattice.

Equations (2) represent the main CA rules that are employed in this model to define the system evolution. They will be applied to the cells with state 2 in order to estimate the 3D stress field within a microstructure that contains many pores. In the simulation of the stress field in a porous body, if we consider a single cellular automaton as an element with the highest symmetry in the volume, then its properties must be compliant with the stress functions (equation 2) for a spherical void. Here, we consider only uniaxial tension as an example, and the result for the stress concentration effect of a single active cellular automaton is shown in Figure $3 \mathrm{a}$ as a $2 \mathrm{D}$ section of the $3 \mathrm{D}$ volume: the single cellular automaton with state 2 , coloured black, corresponds to the smallest size of a void that can be represented in a volume at a given resolution of the CA model. According to the theory of elasticity [51], a void serves as a stress concentrator under tensile load. 
An isolated spherical void of arbitrary radius may be represented by a set of single cellular automatons with state 2, which operate with the rules provided by Eqs. (2), so that the resulting stress field $\sigma_{\text {total }}$ can be calculated as the integral action of all active cells:

$$
\sigma_{\text {total }}=\sum_{i=1}^{N} \sigma_{i}
$$

where $\sigma_{i}$ is the local stress value produced by a cellular automaton with the number $i$ and $N$ is the total number of active cells. The resulting stress concentration factor (SCF), which is the ratio between $\sigma_{\text {total }}$ and the applied tensile stress, is normalised to $N$ to provide a unit value in the regions remote from the void. The result does not depend on the lattice size itself due to the periodical boundary conditions, and the ratio between the defect and lattice sizes also does not have a significant effect. To illustrate this, a comparison between the analytical solution and CA solution is demonstrated in Figure $3 \mathrm{~b}$ for a tensile stress field around a spherical void with the radius of 5 cells. The error is no more than $4 \%$ for the stress concentration factor along the most critical direction, which is orthogonal to the tensile axis.

In the case when more than one pore is located within the microstructure, pore interactions, which are short-range, arise naturally in the CA simulation. As illustrated by the example in Figure $3 b$, pore interaction is significant only within a distance of 10 cells for a spherical pore with a radius of 5 cells. The effect of pore interaction in a microstructure that contains many small pores is obtained by the overlapping stress fields that are created by the active cells in the different pores. If the distance between the interacting pores is small, then their contribution to the sum in Eq. (3) is greater. The total field will show the result of all pore interactions. Such decomposition of a complex problem into sub-problems is common when the final result represents a superposition of the partial solutions [53]. 


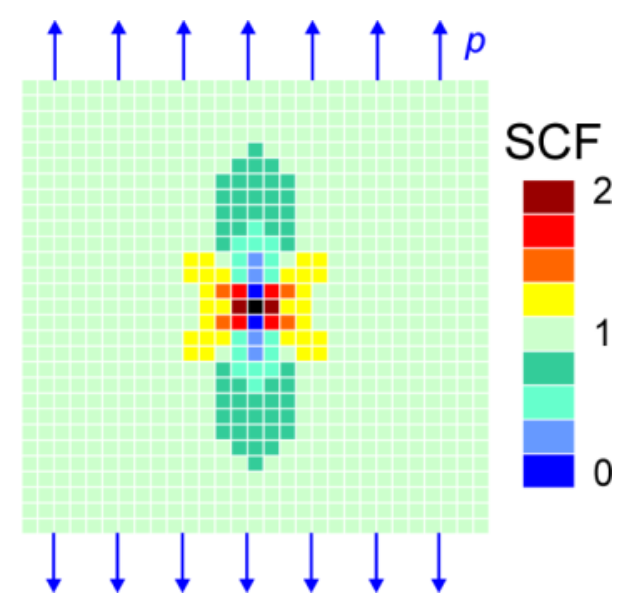

a)

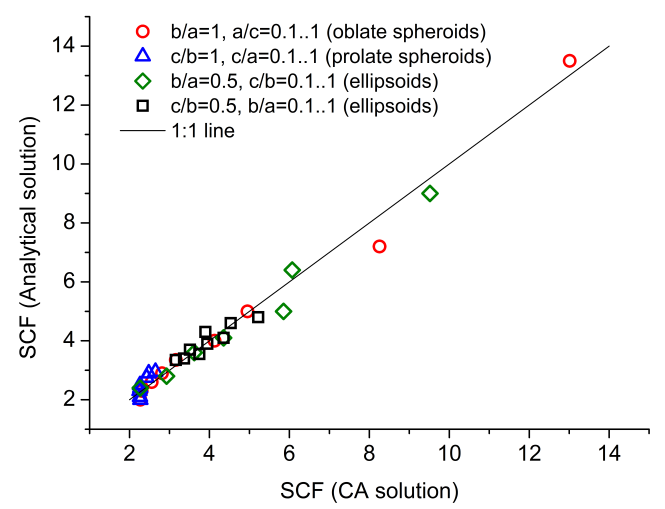

c)

Figure 3 - Cellular automata simulation of stress for uniaxial tension: a) application of the stress functions (equations 2) to one cellular automaton (black cell in the centre) - a 2D $x z$-projection of the 3D maximum stress field is presented; b) 3D maximum principal stress field around an isolated 3D spherical void with the radius of 5 cells; a line profile of the SCF perpendicular to the tensile axis is compared with the analytical solution; c) comparison between CA ellipsoidal correction results and analytical solution for ellipsoidal pores with different elongation; the maximum relative error is no more than $15 \%$.

Linear elastic fields have been assumed here and have been used in the subsequent model calibration, but the model is applicable to non-linear elastic and elastic-plastic fields with corresponding modifications of Eqs. (2) [54]. Using the local elastic properties (i.e. at the cell level), the local stresses obtained in each cell of the matrix then allow calculation of the local strain and strain energy density (SED) values, which may then be used as criteria for fracture. The proposed technique is novel and represents a significant aspect of a fracture algorithm for porous solids, which we describe further in this work. At the same time, the result of the CA simulation must be considered as an approximation, because the technique has the following limitations:

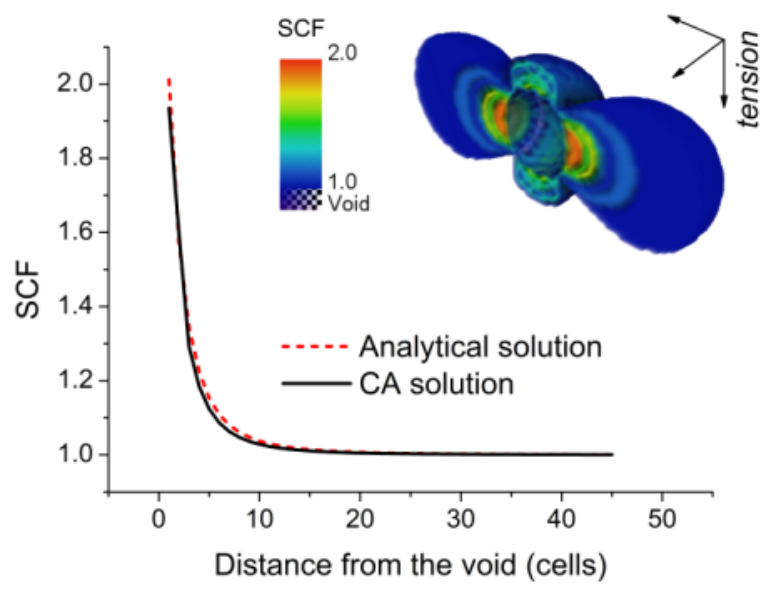

b) 
- no direct algorithm for load redistributions within the CA lattice is involved; the stress field evolution is presented as a sequence of certain states calculated from a current microstructure state and CA rules for fracture development;

- initiated and propagating cracks are considered as a part of the porous structure, i.e. the cells with state 2 ;

- a common ellipsoidal correction is applied to irregular shaped pores and cracks.

The CA approach has advantages when applied to microstructure with pores of complex geometry. Stress calculation using this CA technique is an iterative algorithm in which the total number of iterations equals the number of cells with state 2, i.e. all those that correspond to the pores. As iterations are independent of each other, and the result of the superposition does not depend on the order of computations, the algorithm is parallelised readily.

The CA method requires calibration to the physical problem that is being addressed. The CA method is an approximation, so it is not possible to calibrate to the full stress tensor. However, it is reasonable to calibrate to the maximum principal elastic strain, since it is the dominant contribution to fracture, and the model is currently being applied with linear elastic properties. The stress field calibration of the 3D solution is performed with respect to the analytical solution for a solid with a central spherical void of arbitrary radius [51]. The resulting stress is obtained as a superposition of the stress fields created by unit cells, so it is normalised by the number of these cells. The calibration function obtained between the CA and analytical solutions for the maximum principal stress resulting from uniaxial tension in the $z$-direction has the following form:

$$
\begin{gathered}
y=k_{1}+k_{2} \frac{x}{N} \\
k_{1}=-44.63837-1.23756 N ; \quad k_{2}=k_{1}+1
\end{gathered}
$$

where $N$ is the number of active cells; the coefficients $k_{1}$ and $k_{2}$ are linear functions of $N ; x$ and $y$ are the uncalibrated and calibrated stress concentration factors correspondingly. The calibration is applied to the computed stress field in the cells with state 1; it does not depend on the CA lattice size or void radius.

In case of a non-spherical void, the stress concentration factor changes significantly around the void boundary, especially for an ellipsoidal void with the shape of an oblate spheroid that is orthogonal to the applied stress. The SCF value of an elliptical void exponentially 
decreases with distance from the void, and it is well approximated by the field for a spherical void beyond 2-3 cells from the void. Therefore, for the problem of fracture from a stress concentrator, it is a sufficient approximation if the SCF correction for non-spherical voids is only applied to those cells at its boundary. We have used the analytical solution from [55], which provides the SCF at the boundary of a three-dimensional ellipsoidal cavity. The obtained fit is:

$$
\begin{gathered}
S C F=k_{3}\left(\frac{c}{b}\right)^{k_{4}} \\
k_{3}=2.72956-0.96978\left(\frac{b}{a}\right) \\
k_{4} \approx-0.87939=\mathrm{const}
\end{gathered}
$$

where $a, b$, and $c$ are the semi-axes of the ellipsoid in $x-, y$, and $z$-directions, correspondingly for uniaxial tension applied in the $z$-direction.

This correction function is applied to every boundary cell that is in state 2 . The parameters $a$, $b$, and $c$ are calculated individually for each boundary cell of the void; they are obtained as the number of cells with state 2 in a line across the void from the cell in the $x$-, $y$-, and $z$ directions, and hence are determined by the geometry of the pore to which this cell belongs. Comparison between the results of analytical and CA solutions for ellipsoidal voids with aspect ratios between 0.1 to 1 gives a relative error of no more than $15 \%$ (Figure $3 \mathrm{~b}$ ). Considering that the analytical result is a solution for an infinite volume, the real maximum stress value is lower due to the boundaries, so this error is considered acceptable for the modelling of fracture.

\subsection{Geometrical estimation of the bulk elastic modulus of a porous solid}

Brittle fracture depends on the release of elastic strain energy, so it is necessary to understand the effects of porosity on elastic properties. Experimental data (e.g. for alumina in [9]) show that the bulk value of the Young's modulus depends on the pore geometry as well as the total porosity, and this is also captured by numerical simulations in porous materials (for example $[34,56])$. According to Rice [57], the pore shape effect on elastic properties can be explained by the correspondence of pore geometry with minimum solid areas. Rice showed that several possible mathematical functions could adequately describe the different pore geometries, and considered various calculations of the area around pores of different shapes in a cubic lattice. 
Phani [56] subsequently proposed a semi-empirical general relationship for the dependence of the bulk Young's modulus on porosity:

$$
\frac{E}{E_{0}}=(1-a P)^{n}
$$

where $1 \leq a \leq 3.85$ is the packing geometry factor, $n$ is the pore geometry factor, $E_{0}$ is the Young's modulus for a pore-free material, and $E$ is the Young's modulus for the porous material. Phani's examinations of this relationship for different pore types concluded that, at the same value of $a, n=2.14$ for closed spherical pores and $n=4.12$ for interconnected pores of irregular shapes.

We have used Phani's general relationship (equation 6), as it is capable of providing information for various configurations of pores with different shapes, and have taken the constant value $a=1$ for our simulations of elastic modulus. This satisfies the boundary condition of zero modulus at $100 \%$ porosity. The parameter $n$, which depends on pore shape, can then be extracted geometrically, as follows. The free surface area of all the matrix cells contacting the pores orthogonally to the direction of tension is $A_{\text {surf }}$ (the chosen cells do not have any exposed faces parallel to the direction of tension), and $A_{\text {total }}$ is the total free surface area of all the 'solid' cells surrounding the pore (Figure 4a). The figure shows a single plane, and these areas are summed for the series of planes that is parallel to the direction of tension. Then, we assume that

$$
n=\left(\frac{A_{\text {surf }}}{A_{\text {total }}}\right)^{-k}
$$

where $k=1 / 2$ is the topological factor that is dictated by the cell's cubic shape in the CA lattice. 


\section{tension}

a)


b) 


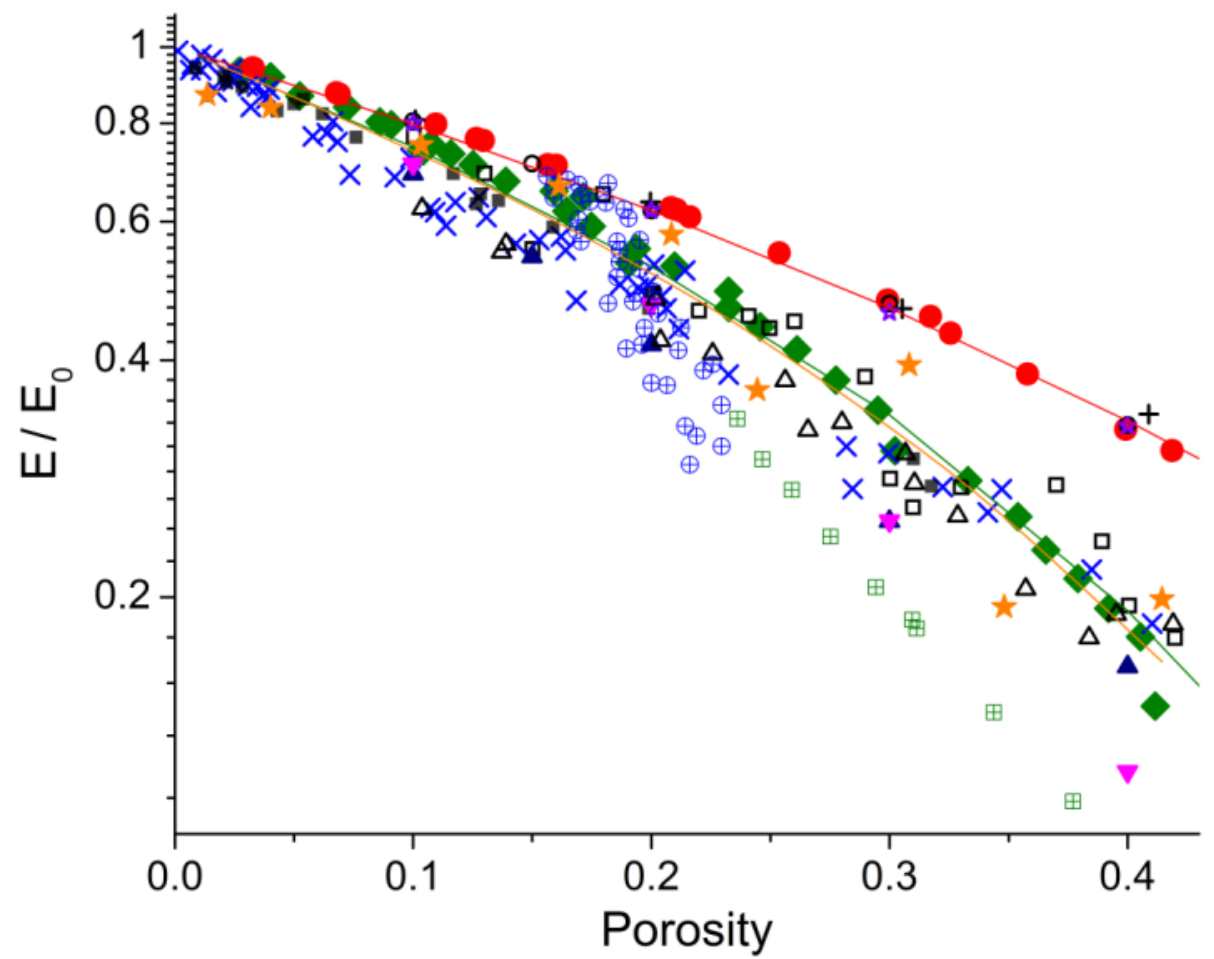

Model:

- Arbitrary shaped pores I (our model)

Experiment:

*-Arbitrary shaped pores II (our model)

- $\mathrm{ZrO}_{2}[$ Smolin]

- Spherical pores (our model)

+ Alumina [Coble]

- Spherical pores [Smolin]

$\times$ Alumina [Knudsen]

- Spherical solids [Smolin]

* Spherical pores [Roberts]

- $\mathrm{MgAl}_{2} \mathrm{O}_{4}$ [Porter]

$\oplus \quad$ Graphite [Pickup]

$\checkmark$ Spherical solids [Roberts]

c)

$\Delta$ Image-based FE-model: Graphite [Berre]

Figure 4 - Computation of the Young's modulus value in a porous solid: a) schematic for estimation of the pore geometry factor in a cube shaped lattice; b) results for the bulk Young's modulus behaviour in microstructures with different pore shapes compared with Phani's relationships [56]; c) comparison with experimental and modelling data from literature: models from Smolin [58], Roberts [59] and Berre [60], and experimental data taken from Smolin [47], Coble [9], Knudsen [61], Porter [62], Pickup [63], and Imai [64]

To test this relationship, we have examined, for different degrees of porosity, 28 synthetic microstructures with ellipsoidal pores, 32 microstructures with spherical pores and 63 microstructures with arbitrary shaped pores created with 2 different distributions with a basis of an actual pore size distribution measured in a virgin nuclear graphite [65]. All synthetic microstructure were simulated in a cubic volume of $100 \times 100 \times 100$ cells with periodic boundaries and random pore distribution within the volume. In each case, the parameter $n$ was obtained directly from all the pores of the microstructure and the elastic modulus calculated using equations 6 and 7. The result is shown in Figure 4b. Unlike Phani's 
calculations, the parameter $n$ that is obtained directly from the pores is not constant in our simulations. This is due to the allowed overlapping of pores; $n$ varies from 1.96 to 2.24 for spherical and ellipsoidal pores, and from 2.74 to 4.52 for the arbitrary pores. The results for the microstructures with spherical and ellipsoidal pores in our simulations have similar behaviour, because both spheres and ellipsoids are topologically identical in the cubic lattice of our model. These data correspond very well to Phani's curves for closed spherical pores (with $n=2.14$ ). The simulated data points for arbitrary pores agree well with Phani's curve for interconnected irregular pores (with $n=4.12$ ).

Our results for elastic modulus are compared with other models and experimental data in Figure 4c. They are in full agreement with the modelling results for spherical pores of Roberts [59], who used the FE method, and Smolin [58], who used the finite difference scheme. Roberts [59] and Smolin [58] also simulated synthetic microstructures created by overlapping solids (i.e. irregular shaped pores) and obtained similar results. The moduli for the arbitrary microstructures presented in this work for irregular shaped pores are close to the experimental data for such microstructures, except for the data from thermally oxidised graphite. All models for elastic modulus show sensitivity to the geometry of the pores, and full validation of any model will require experimental data from microstructures in which the pores have been fully characterised. This has been done, for instance, for thermally oxidised graphite by Berre [60], using a multi-phase FE model that assigned mechanical properties using the local graphite density that was obtained from the measured X-ray attenuation. That model also overestimated the experimental data of Pickup [63] and Imai [64] for thermally oxidised graphite. This may be due to differences in the microstructures of the graphite samples that were tomographed by Berre and those tested by Pickup and Imai, as thermal oxidation is quite sensitive to graphite purity, microstructure and oxidation conditions [64]. The simulation results of Berre are quite close to the other modelling simulations for arbitrary shaped pores.

The results for our model for the bulk Young's modulus in linear elastic porous microstructures are therefore quite consistent with published models and data. Having a practical and flexible method to calculate the elastic Young's modulus of a volume with an arbitrary distribution of pores, and hence its strain energy, a fracture algorithm can now be developed. A simplifying assumption is made of constant Poisson's ratio with increasing porosity. This is judged to be reasonable, since as shown in [59], the Young's modulus of a porous body is practically independent of the solid Poisson's ratio. 


\subsection{Fracture algorithm}

Fracture development in a brittle porous material is a complex, competitive process; the stress field created in a porous microstructure may allow simultaneous crack initiation in different regions of the volume, and depending on the pore geometry, cracks may also be deflected by pores, for instance [66]. A fracture initiation and propagation algorithm has been developed on the basis of the CA technique, which recalculates the stress field at each stage of the damage process. The fracture model supports multi-crack initiation and fracture propagation.

The physical parameters used in the model have been separated into global and local parameters. The local state $\omega_{i}$ of each cell $(i)$ associated with a solid material can be schematically described by the following parameters:

$$
\omega_{i}=\left\{\sigma_{i}, \varepsilon_{i}, U_{i}, E, v\right\}
$$

where $\sigma_{i}, \varepsilon_{i}, U_{i}$ are the local values of the stress, strain and strain energy; $E$ is local Young's modulus; and $v$ is Poisson's ratio. These are the properties of the material at the length scale of the CA cell.

The global state $\Omega$ of the model is defined as

$$
\Omega=\left\{\sigma_{a p p}, \varepsilon_{a p p}, U_{t o t a l}, E_{b u l k}, v\right\}
$$

where $\sigma_{a p p}$ and $\varepsilon_{a p p}$ are the applied stress and strain, on the basis of which the distribution of the local stresses/strains is calculated in each cell of the microstructure. The global value of elastic energy $U_{\text {total }}$ of the system is equal to the sum of the local values $U_{i}$ in each cell of the lattice. The important property of this parameter is that it can be defined independently on either local or global levels through the corresponding local or global values of the stress and Young's modulus. These are provided by the homogenisation procedure at each step of the fracture algorithm. For Poisson's ratio, we have used the value $v=0.21$, which is constant on both local and global levels in this consideration of the model, although separate values could be defined.

The separation between the characteristics for the local and global parameters allows the fracture algorithm to be supplemented with a feedback loop that calculates the effects of damage on the applied load; a fixed boundary displacement is applied and damage that increases the porosity will decrease the bulk elastic modulus. This is realised on the basis of the energy balance of the simulated system: 


$$
\frac{d U_{t o t a l}}{d n}=\frac{d \Pi}{d n}+\frac{d W}{d n}
$$

where $n$ is the number of damaged cells in the lattice, $\Pi$ is the potential energy (i.e. the elastic strain energy), and $W$ is the work required to produce new damaged cells.

The fracture algorithm includes the following stages:

- The initial calculation of stresses in the porous microstructure using the CA algorithms described above, and its elastic modulus, which is calculated directly from the pore population.

- Each cell that corresponds to the solid fraction is tested for compliance with the fracture criterion; this may be defined arbitrarily as a critical stress, strain, strain energy (SE), or SE release rate.

- If the chosen fracture criterion is satisfied in any cell, then this cell is identified as damaged and changes state from inactive to active. If there is no damage at the current iteration, then the applied load/displacement increases with a given step and the algorithm starts from the beginning.

- The total energy of the system is recalculated, taking into account the energy costs such as elastic energy release and energy for surface creation, which are properties of the cell.

- The change in bulk Young's modulus due to the addition of the damaged cells to the porosity leads to recalculation of the applied stress and the new value of the total energy to satisfy the energy balance according to Eq. (10).

- After computing the new distribution of the local stresses, the fracture criterion of all cells is then checked during the next iteration of the algorithm.

The simulation continues as damage propagates, until convergence is reached in equilibrium with the applied displacement. In the fracture simulations, the failure stress has been defined as the applied stress above which the damage propagation is unstable (i.e. catastrophic). We simulate the damage development on the basis of the approximation for the stress field around a void instead of a crack. The ellipsoidal correction used in the model operates in the neighbourhood of the voids, and since the stress field decays dramatically with increasing distance, the decision about fracture in the cell is dominated by the state of its nearest neighbours, which provide a local SCF value. 
The appropriateness of the fracture algorithm can be shown by the simulation of the propagation of a single 'penny-shaped' 'crack' (one cell thick) located in a homogeneous material with a uniaxial tensile stress, $\sigma$, applied orthogonally to the defect's plane. The chosen fracture criterion was a nominal strain energy release rate equal to $2 \mathrm{~kJ} / \mathrm{m}^{2}$. This requires actual dimensions to be considered for the cells, so the initial crack radius, $a$, varies from 0.01 to $0.4 \mathrm{~mm}$, which corresponds to 1 to 40 cells in the lattice of $100^{3}$ cells. In the simulation, the crack-like void grew in a plane orthogonal to the stress. The failure stress is obtained as the stress at which propagation of the defect becomes unstable. The obtained dependence between the strength and defect size presented in Figure 5a has a best fit of $a^{-0.49}$. This is in good agreement with the classical linear elastic fracture mechanics $a^{-1 / 2}$ relationship for this crack geometry [67], given that a penny-shaped crack is an extreme form of an elliptical defect, and the SCF correction is applied only to the cells at the edge of the void.

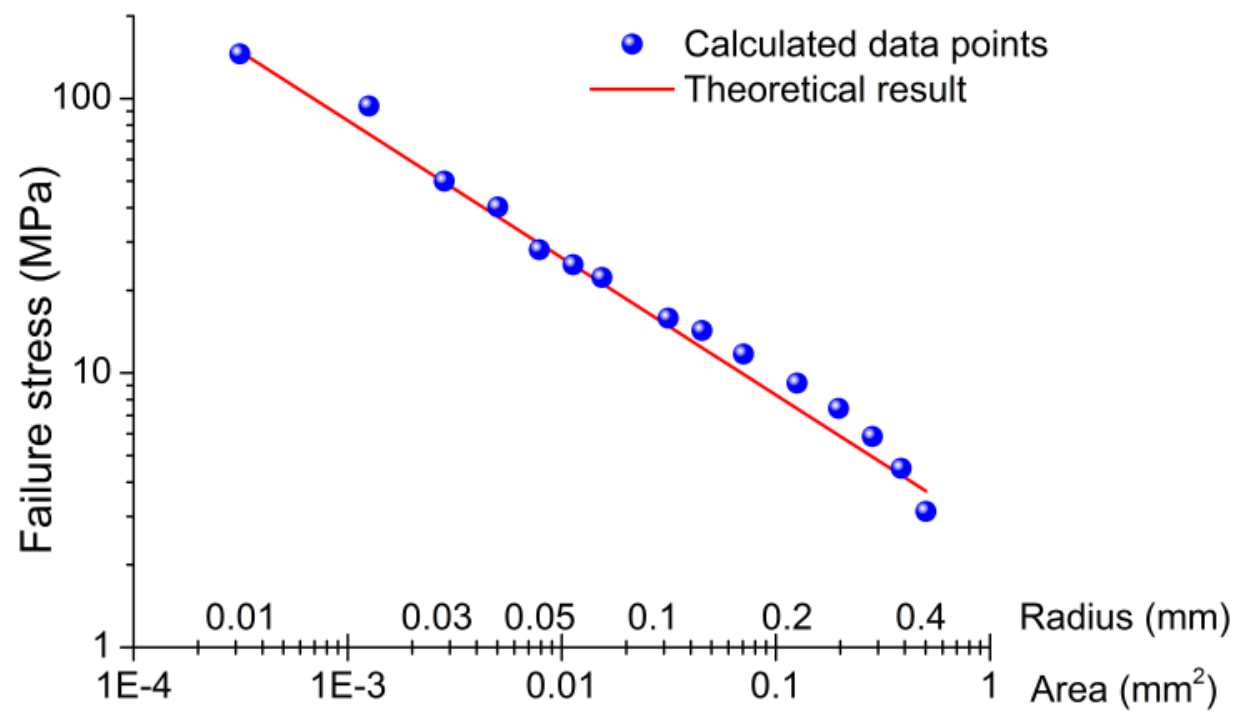

a) 




Model:

- Arbitrary shaped pores I (our model)
$\star$ Arbitrary shaped pores II (our model)
- Spherical pores (our model)
$\circ \quad$ Spherical pores [Smolin]
$-\quad$ Spherical solids [Smolin]
$\Delta \quad$ Image-based FE-model: Graphite [Berre]

Experiment:

- $\mathrm{ZrO}_{2}[$ Smolin]

$\nabla \quad$ Plaster [Coble]

+ Alumina [Coble]

- $\mathrm{ZrO}_{2}[$ Kalatur $]$

$\oplus \quad$ Graphite [Berre]

b)

Figure 5 - Validation of the model against known dependencies: a) simulated data for penny shaped defect with different initial radii. ( 1 cell is $0.01 \mathrm{~mm}$ ). The critical fracture energy is $2 \mathrm{~kJ} / \mathrm{m}^{2}$, Young's modulus is $10.85 \mathrm{GPa}$. The data are compared with the theoretical results of the linear elastic fracture mechanics analytical solution for a sharp crack; b) dependence of the fracture strength on total porosity in a comparison with other models from Smolin [58], Berre [24], and experimental data from Smolin [47], Coble [9], Kalatur [68], and Berre [60].

The fracture simulation was also tested for the model microstructures with different amounts of spherical pores and arbitrary pore shapes (using the same two distributions examined for elastic modulus), and the results compared with other models and data in the literature (Figure $5 \mathrm{~b}$ ). The strength-porosity simulations for microstructures with arbitrary pores differ significantly from the curves for microstructures with spherical pores, showing that microstructures with a different geometry and spatial distribution of pores present a different dependency of strength on the total porosity. This is also illustrated by the different strengths 
obtained for the two arbitrary-shaped pore populations, which had quite similar elastic modulus-porosity relationships (Figure $4 b$ ). In an FE simulation of the effects of the complex porosity of oxidised graphite [60], which directly used X-ray tomographic microstructure data to develop a multi-phase 'smeared crack' solution, good agreement between model and experimental data was achieved for tensile (flexural) strength; unlike the study of elastic modulus, the tested oxidised graphites had the same microstructures as used for the model. The FE model was limited, however, to just $40 \times 40 \times 40$ elements $\left(0.75 \mathrm{~mm}^{3}\right)$ due to computational restrictions. The finite difference model of Smolin [58] also achieved quite good simulation of the compressive strength of Zirconia, a porous solid with complex pores that was represented by overlapping spherical solids. It is clear, however, that the behaviours of zirconia ceramics can differ; this is likely to be due to microstructural differences in the pore geometry and heterogeneity of distribution. The CA simulations for the strength of a solid with arbitrary pores, particularly from distribution II, tend to agree with the experimental data from Coble [9], and Kalatur [68] for flexural strength of alumina and compressive strength of zirconia. The pores in Coble's study are uniformly distributed, but have irregular shapes, so it is reasonable that the strength trend is closer to the simulations for arbitrary pores than spherical pores. In summary, it is important that strength simulations in porous brittle solids should accurately represent the pore geometry, and this can be done using the CA method.

\subsection{Multi-scale CA model for nuclear graphite}

Having demonstrated the performance of the CA simulation for fracture and elastic properties, we now apply it to prediction of the mechanical properties of radiolytically oxidised nuclear graphite. This requires a multi-scale model due to the heterogeneous structure of filler and matrix, and the different development of fine porosity in these with oxidation. The multi-scale model has the size of $5 \times 5 \times 5 \mathrm{~mm}^{3}$, realised at the large scale in a lattice of $100 \times 100 \times 100$ cells. This volume contains large pores with arbitrary shapes and sizes between 0.1 to $1 \mathrm{~mm}$. Spherical filler particles have been inserted randomly according to the size distributions in Figure $2 \mathrm{c}$ and filler fractions in Figure 2a that were observed on the basis of tomography data for the corresponding grade of oxidation.

The large pore fraction introduced into the larger scale model is currently assumed to be constant equal to $6.5 \% \pm 0.5 \%$ and is distributed in the volume from a known size distribution; this is the porosity of a typical virgin non-oxidised graphite and was measured using laboratory tomography data of a volume of $13.5 \times 13.5 \times 12.2 \mathrm{~mm}$ at a voxel size of 10 
$\mu \mathrm{m}$. By setting a constant fraction of the large pores for oxidised microstructures, we assume that the most significant changes occur within the microstructure's small scale porosity, as observed experimentally [8, 69]. The discrete model requires re-binning of the pores in the 3D data that represent the microstructures, which leads to a loss of fine pores during the binning, which is shown in Figure 6. However, the finest pores that are lost in the binning are accounted for in the total porosity of the bulk microstructure, obtained either from the density or measured weight loss. The total porosity is used to calculate the bulk modulus. The binning is necessary for the simulations, because the model is restricted by the $100 \mathrm{x} 100 \mathrm{x}$ 100 lattice size used for microstructure representation.

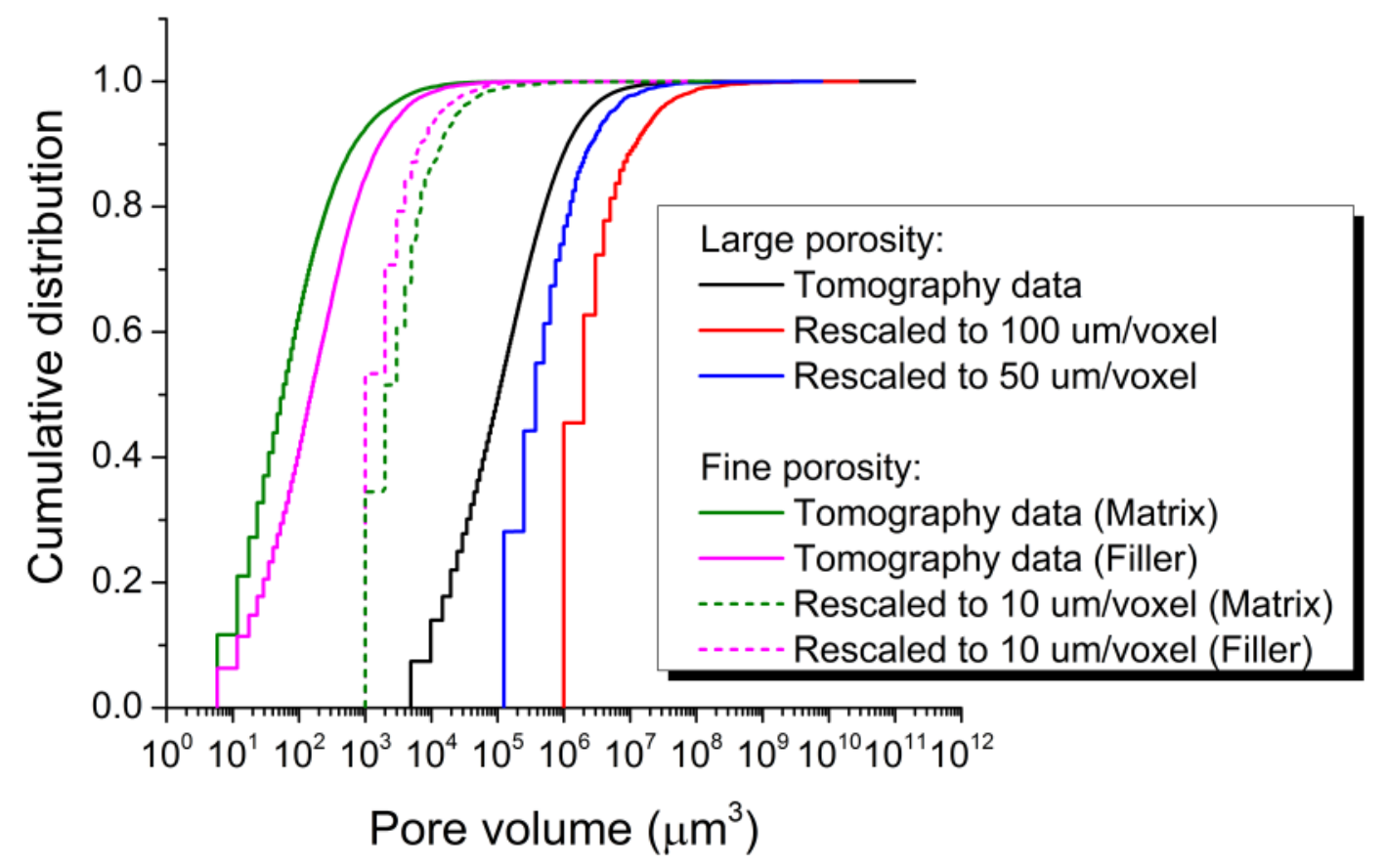

Figure 6 - Large and fine pore size distributions for original tomography data and rescaled (i.e. binned) data.

The matrix fraction is not measured, and so is calculated as the difference between the full volume and the fractions of the filler and the large pores. To test whether this approximation is appropriate, the total porosity $(T P)$ can be determined using either the calculated fractions as

$$
T P=F P^{*} F+M P^{*} M+L
$$

or the weight loss values: 


$$
T P=1-\frac{\text { Virgin density }}{\text { Crystal density }} *(1-W)
$$

where $F P$ and $M P$ are the average filler and matrix porosities correspondingly at that weight loss (see Figure 2b); $F, M$ and $L$ are the filler, matrix and large pores fractions correspondingly; $W$ is the weight loss; Virgin density is the graphite density in its virgin state before oxidation, which is $1.805 \mathrm{~g} / \mathrm{cm}^{3}$ for this nuclear graphite [70]; Crystal density $=2.26$ $\mathrm{g} / \mathrm{cm}^{3}$ is the density of a graphite single crystal. As can be seen from Figure 7, both methods give approximately the same result. This supports the assumption that the regions of microstructure that are not recognisable as filler particles may be represented as matrix, and that the large pores do not contribute significantly to the change in total porosity. An example of a large scale model of filler and porous structures for the sample with $68.2 \%$ weight loss is presented in Figure $7 \mathrm{~b}$.

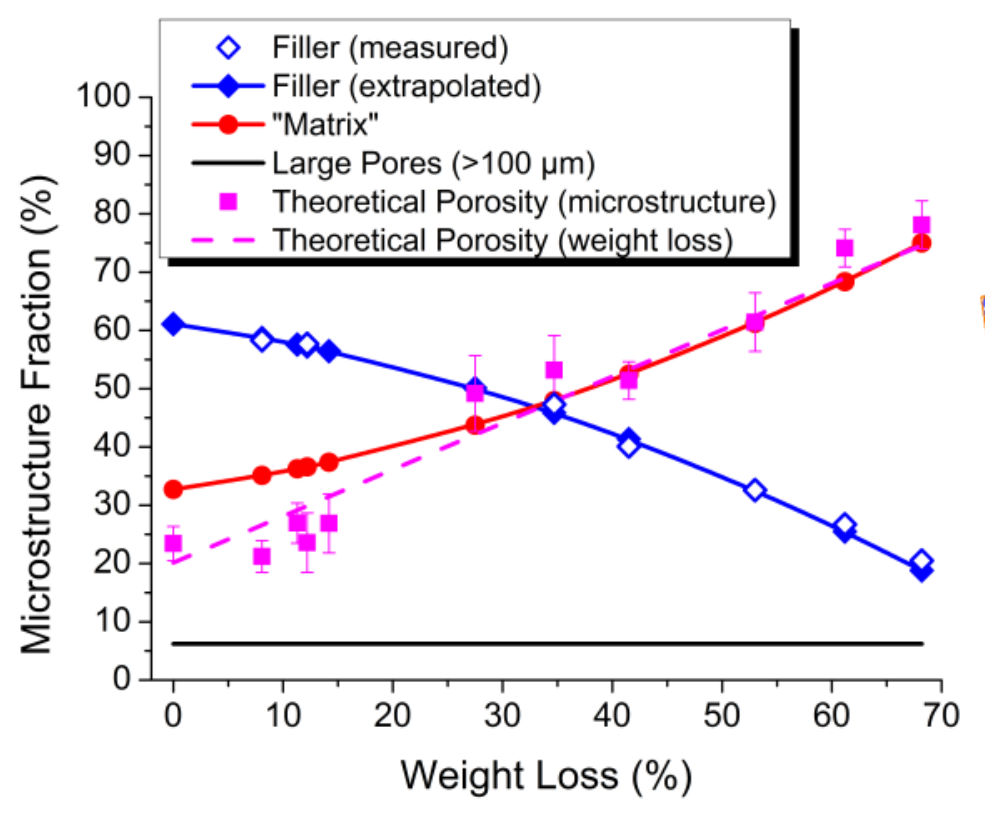

a)

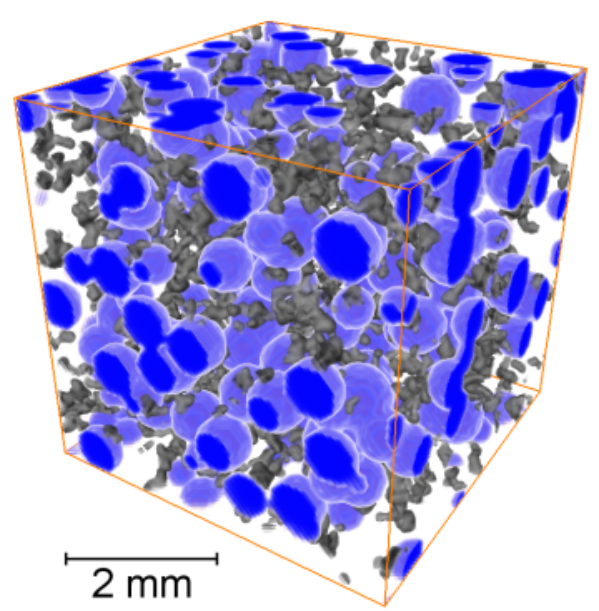

b)

Figure 7 - Fractions of filler, matrix and large pores used in the model of HPB graphite (a) and a large scale model for $\mathbf{6 8 . 2 \%}$ weight loss (b). Filler is coloured in blue, large pores are grey and the matrix is transparent. A virgin density of $\mathbf{1 . 8 0 5}$ is used for the total porosity calculation according to Eq. (11) with the use of measured filler, matrix and pore fractions and Eq. (12), which uses experimentally determined weight loss. The extrapolated filler particle fractions are obtained at different weight loss from the fit in Figure 2a.

The most significant changes caused by radiolytic oxidation are due to the increasing porosity content, and it has been shown by experimental data (e.g. [71]) that neutron irradiation does not have a significant effect on the pore structure and mechanical properties. Fast neutron 
irradiation does affect modulus and strength independently of oxidation [3]. However, for the selected samples that all have received quite high neutron dose (Table 1), the relative effects of neutron dose on strength and modulus, through their effect on the structure factor, are small compared to the effects of the weight loss from oxidation [5], and so can be neglected. The effect of weight loss is traditionally described as an exponential function of fractional weight loss with different coefficients for elastic modulus $\left(W_{E}\right)$ and strength $\left(W_{S}\right)$ [72]. It is noted in reference [72] that there is a limit of applicability for the exponential estimation at $33-45 \%$ of weight loss, depending on the neutron dose.

The effect of total porosity on elastic modulus is described by the weight loss term $W(E)_{C A}$ for the large scale developed model. On the basis that the bulk microstructures contain a large number of pores of complex shape, it is assumed that this weight loss term may be obtained using the procedure for geometrical computation of elastic modulus that was described above for arbitrary shaped pores as a ratio between bulk modulus, $E$, and pore-free modulus, $E_{0}$. It is represented by a power function [56]:

$$
W(E)_{C A}=\frac{E}{E_{0}}=(1-T P)^{4.12}
$$

where $T P$ is the total porosity of the samples.

At the fine scale of the multi-scale model, the Young's modulus values calculated for the individual sub-volumes of filler particles and matrix using Eqs. 6 and 7, and the fracture algorithm presented in Section 3.4 has been used for the simulations of tensile strength within the microstructures of filler and matrix. Uniaxial load was applied to each of the sub-volumes in three orthogonal directions with a fixed displacement condition; a nominal strain energy release rate of $200 \mathrm{~J} / \mathrm{m}^{2}$ was used as the fracture criterion. The outputs of these small scale models are statistical distributions of strength and elastic modulus for filler particles and matrix, as a function of the weight loss term.

The heterogeneous properties obtained by the small scale models of filler and matrix were then introduced to the cells of the large scale model. At this longer length scale, a critical strain is used as a fracture criterion, and is computed for the cells associated with the filler and matrix as the ratio between the critical stress and elastic modulus; these values are randomly extracted from the corresponding coupled distributions that are provided by the individual small scale models for filler and matrix. On this basis, tensile stress-strain curves 
have been obtained for each microstructure. These have been compared with available experimental data for virgin and irradiated oxidised graphite.

\section{Results}

The Young's modulus values calculated for the individual sub-volumes of filler particles and matrix are plotted as a function of the porosity of each sub-volume in Figure 8a, which shows that both filler and matrix in the oxidised microstructures can be approximated by a function with the power of 4 . The consistency of these directly evaluated values with the weight loss term of Eq. (4) demonstrates the generality of this function for microstructures with complex pores. The relative changes in the mean elastic modulus predicted for the filler and matrix in oxidised graphite are summarised in Figure $8 \mathrm{~b}$ as a function of the bulk weight loss. This clearly shows the greater effect of oxidation on the matrix compared to the filler, which arises from the preferential oxidation of the matrix compared to filler that is apparent in Figure $2 b$.

The tensile strength values predicted by the small scale models of the matrix and filler subvolumes are presented in Figure 8c as a function of their porosity. Quite different trends for the strength of the matrix and filler particles are observed. This arises from their different pore structures and the organisation of porosity within the individual sub-volumes, to which the elastic modulus is less sensitive. The mean data are presented as a function of the bulk weight loss of the graphite microstructure in Figure $8 \mathrm{~d}$. The data are normalised by the constant $\sigma_{o}$, which had a value of $138 \mathrm{MPa}$. This was obtained by extrapolation of the simulations for the oxidised matrix to zero porosity, and its value is dependent on an assumed work of fracture of $200 \mathrm{Jm}^{-2}$, which is taken from reference [1]. The mean matrix strength decays exponentially with increasing oxidation (Figure 8d), but the mean strength of the filler particles may be considered as effectively constant. Quite scattered strengths are predicted for the filler particles (Figure 8c), but these are generally higher than the matrix strengths, so the matrix strength can be expected to dominate the failure of oxidised graphite. 

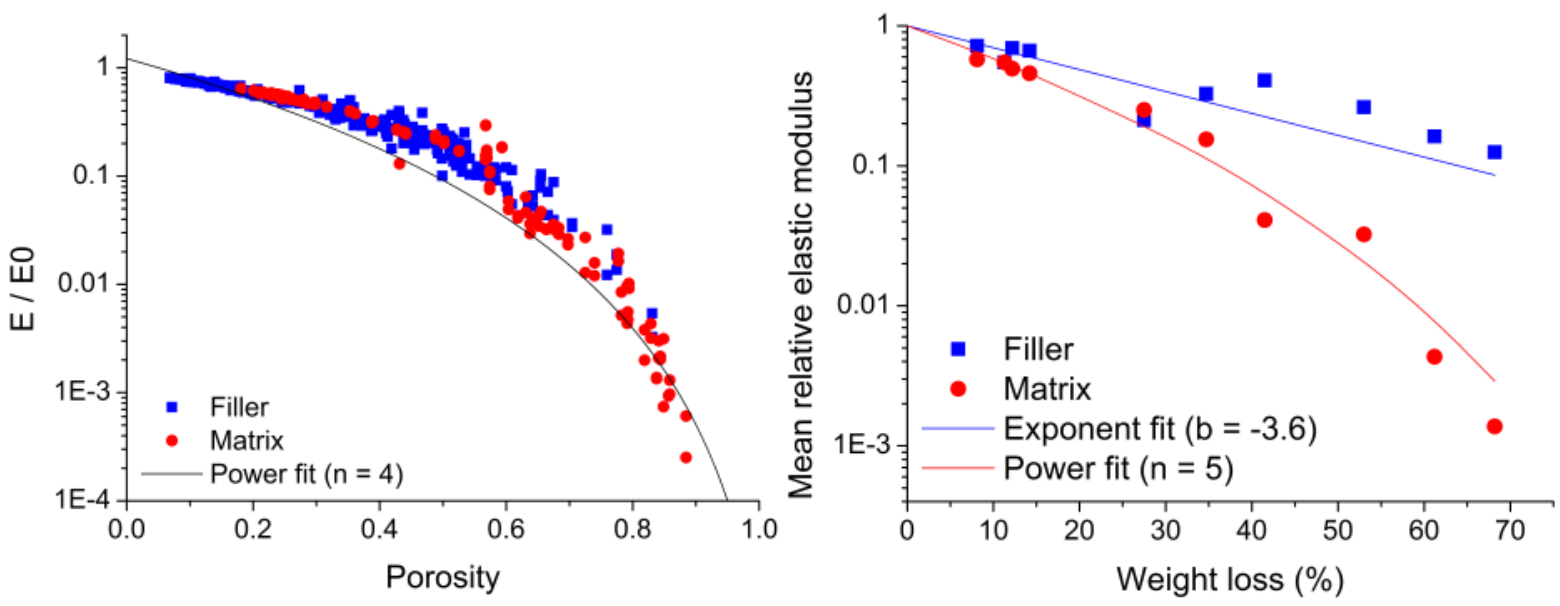

a)

b)
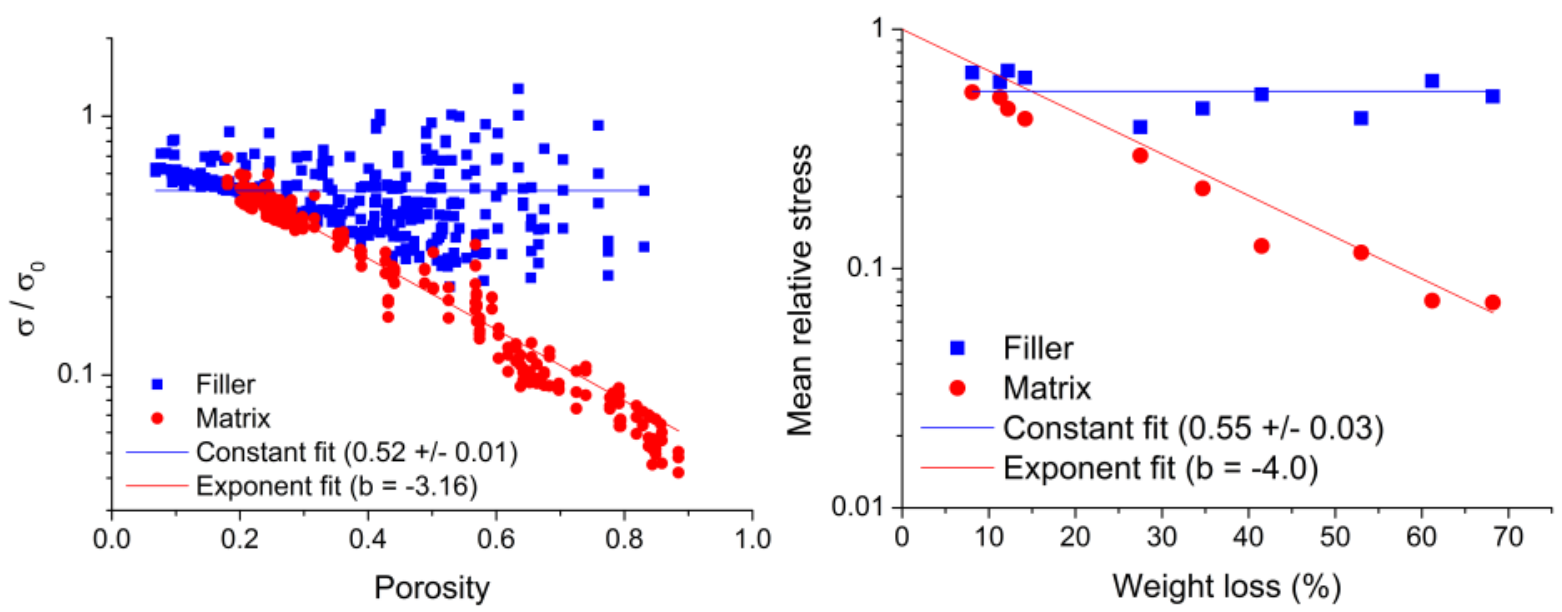

c)

d)

Figure 8 - Predicted degradation of elastic modulus $(a, b)$ and failure stress values $(c, d)$ of the filler and matrix in radiolytically oxidised HPB graphite. Mean values of elastic modulus (b) and failure stress (d) for oxidised microstructures are presented in comparison with the corresponding fitting curves. Elastic modulus (b) is normalised to $E_{0}=100 \mathrm{GPa}$, stress (d) is normalised to $\sigma_{o}=138 \mathrm{MPa}$, obtained by extrapolation of simulations for irradiated oxidised matrix shown in (d) for both filler and matrix. 




a)

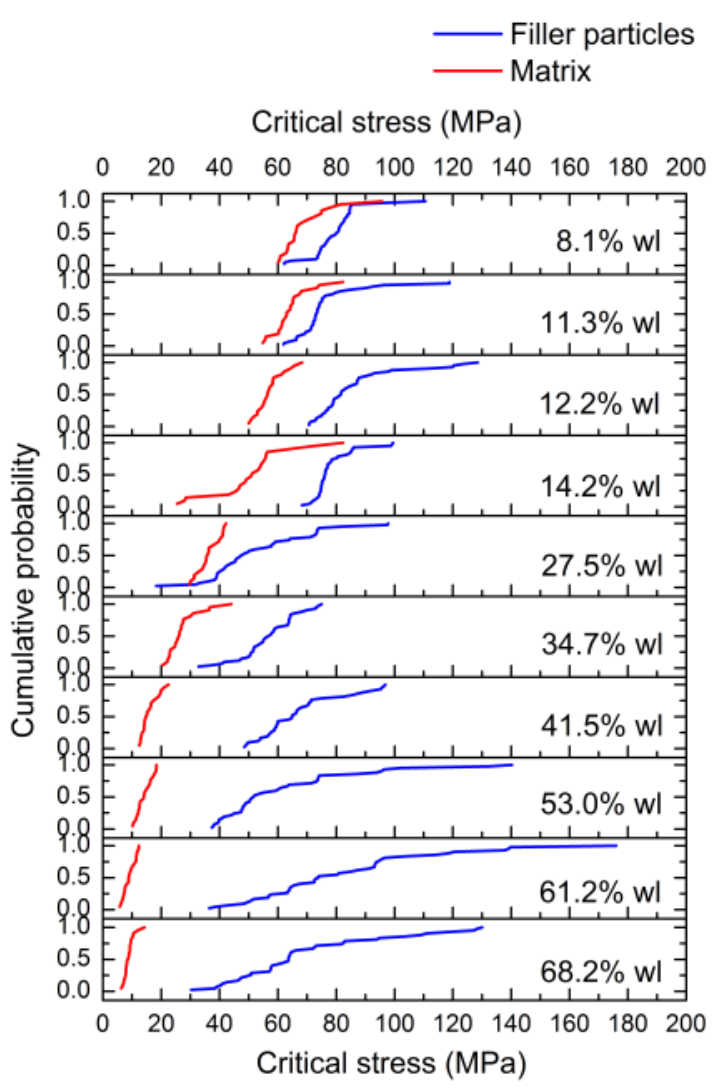

b)

Figure 9 - Results of the small scale simulations obtained for elastic modulus (a) and critical failure stress values (b) of the filler and matrix in radiolytically oxidised HPB graphite.

The distributions of strength and local elastic modulus obtained from the CA small scale model were assigned to the corresponding cells of filler and matrix in the large scale model. The critical strain, obtained on the basis of the coupled values from these two distributions (i.e. coupling of values from the same simulated sub-volumes), was used as the fracture criterion. The small scale simulations were performed with a work of fracture of $200 \mathrm{Jm}^{-2}$ and also $400 \mathrm{Jm}^{-2}$ to investigate sensitivity to this parameter, and the simulated stress-strain curves at $200 \mathrm{Jm}^{-2}$ are presented in Figure 10a for the range of weight losses of 8.1-68.2\%.

There are currently no experimental strength data available for the individually characterised samples at the given weight loss, so the predicted strength values are shown in Figure 10b in comparison with available experimental data for radiolytically oxidised samples of this graphite grade and also for other similar grades from various AGR stations. The tensile strengths were, in some cases, obtained by the correction of measured flexural strength data in specimens of comparable size, using the method described in [73] (18 pairs of flexural and 
tensile strength values at approximately the same weight loss values in the available experimental datasets were used to calibrate the correction). Exponential 'Knudsen' curves from earlier data fits to radiolytically oxidised Gilsocarbon graphite by Kelly [72] with the pre-exponential factor of 4 for strength and -3.6 for elastic modulus in Figure $10 \mathrm{~b}$ and c are computed with the same pore-free values of the simulations. The multi-scale model demonstrates a quite good agreement with the experimental data for tensile strength of the general population of radiolytically oxidised graphites, except for the strength at low weight loss.

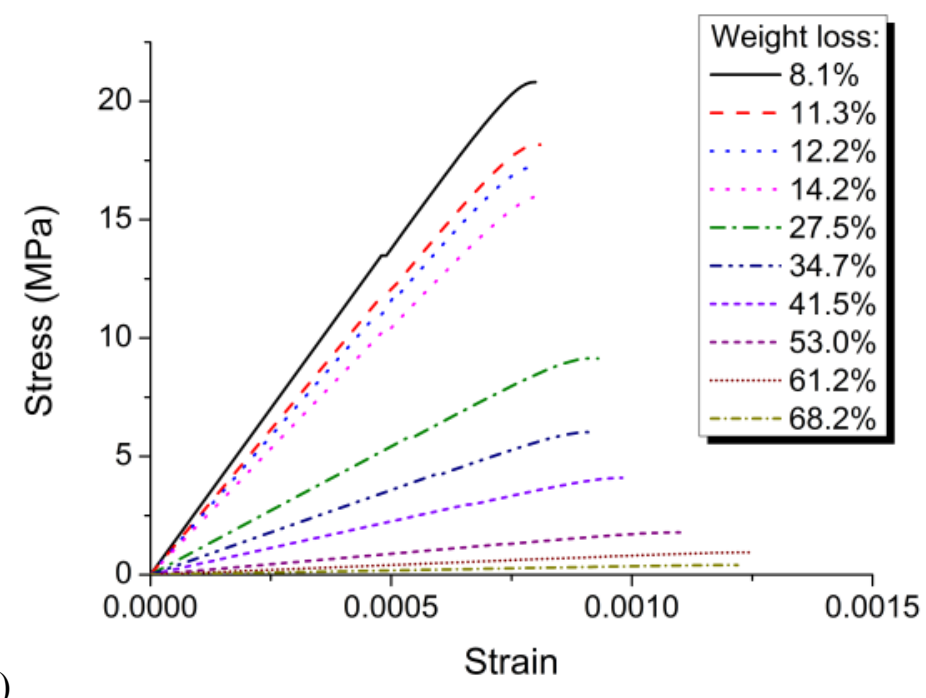

a)



b)



c)

Figure 10 - Results of the large scale simulations: a) simulated tensile stress-strain curves for different weight loss; and effect of weight loss on b) tensile strength and c) elastic modulus for the radiolytically oxidised HPB graphite in comparison with experimental data for different graphite grades. 


\section{Discussion}

The comparison between filler and matrix behaviours for the radiolytically oxidised samples shows that the changes in the matrix microstructure are more significant than in the filler (Figure 8), and that these have a greater effect with increasing weight loss. The mean values of simulated Young's modulus and tensile strength (i.e. at 50\% probability) for filler and matrix in oxidised microstructures may be compared with Kelly's exponential relationship [72]; this is an established approximation obtained from experiments performed on oxidised graphite up to a weight loss of around $30 \%$. The normalised values of the elastic modulus for filler and matrix both follow Kelly's relationship (exponent -3.6) up to 30\% weight loss (Figure $8 \mathrm{~b}$ ). Above that, the matrix degradation is predicted to increase relatively for highly oxidised microstructures while the filler simulations continue to follow the exponential relationship. Young's modulus values computed for the large scale models are also compared with the Kelly's relationship (Figure 10c) and available experimental data. As seen, both overestimate elastic modulus values in the low weight loss region. However, our computations have a better agreement with experimental data in the region of high weight loss. The agreement at low weight loss is not ideal, but the current model does not include the non-linear influence of the fast neutron dose on the pore-free modulus through its effect on the structure factor. As oxidation tends to increase in proportion with irradiation dose, a reduced effect of fast neutron irradiation could reduce the modulus by up to a factor of 2 for some low dose samples $[4,5]$.

The prediction of the tensile strength obtained with the developed multi-scale model for radiolytically oxidised graphite is in agreement with available experimental data (Figure 10b) in the range of medium and high weight loss values, however, the simulations underestimate the strength in a low weight loss region in the case where $200 \mathrm{~J} / \mathrm{m}^{2}$ is used as a fracture criterion. This may be due partly to overestimation of the elastic modulus, noted above, from the assumption of a constant irradiation effect on $E_{0}$. The model is derived purely from microstructure characterisation and the semi-empirical fitting of two general parameters; the pore-free modulus and the pore-free strength, which depends on the work of fracture of porefree graphite. There is a weak effect of the work of fracture, and the simulations with a higher work of fracture value give a good fit to experimental data in the region of medium weight losses (up to $40 \%$ ), but overestimate strengths in the high weight loss region. This effect could be explained if there was a lower work of fracture at higher weight loss, which might 
occur due to oxidation that was below the resolution of the tomographs, as has been observed in the anisotropic PGA graphite [74].

Multiple runs of a large scale model with different microstructures of a chosen filler/matrix/pores composition are possible due to the computational efficiency of the CA method, and this allows the potential stochastic effects of microstructure heterogeneity to be investigated. Analysis of microstructures with the same ratio of matrix to filler at the same weight loss, but with different arrangements, found less than $2 \%$ variation in strength. The modulus, which depends on the total porosity, was unaffected also. However, graphite is a coarse heterogeneous material, and within small volumes local variations might be expected in the filler to matrix ratio. To assess the possible effect of the filler to matrix ratio, the properties of three microstructures with the different filler and matrix ratios has been simulated for each weight loss value. Changing the ratio of filler to matrix has an effect on the average porosity (see Eq. (2)), and the purpose of this analysis is to estimate the possible effect of differences due to unknown (i.e. not measured) changes in filler to matrix ratio. This might occur, for example, in the testing of small samples extracted from a larger volume of graphite in which the average weight loss has been determined. The effect is illustrated by using a gradient $(g)$ that describes the rate of change with filler/matrix ratio of the property from the expected value; at each nominal weight loss, the strength gradient was calculated as a ratio of the strength change $(\mathrm{d} S)$ to the filler fraction change $(d F F)$ relative to the expected values of the strength $\left(S_{\text {exp }}\right)$ and filler fraction $\left(F F_{\text {exp }}\right)$ at that weight loss:

$$
g=\frac{d S}{d F F} ; \quad d S=\frac{S-S_{\text {exp }}}{S_{\text {exp }}} ; \quad d F F=\frac{F F-F F_{\text {exp }}}{F F_{\text {exp }}}
$$

The elastic modulus gradient was calculated in the same manner. Using the parameters for the characterised radiolytically oxidised graphite, both strength and elastic modulus have similar behaviour (Figure 11), and demonstrate a greater response of highly oxidised microstructures to a change in filler/matrix content. This suggests that, in the absence of other factors, the measured mechanical properties are more sensitive at high weight loss to local variations in filler to matrix ratio. For instance, at approximately $40 \%$ weight loss, a variation of $8 \%$ in the filler to matrix ratio from the expected value of $40 \%$ filler to $48 \%$, would be predicted to change the strength by $25 \%$. The increased variability measured for small test specimens due to variations in the filler to matrix ratio might be described empirically using Weibull statistics [75], which could then be applied in a probabilistic fracture model of the graphite components' engineering integrity [76]. However, the underlying factors that control 
size dependence would not simply be the frequency distribution of defect size that is assumed in the Weibull analysis, and this could limit its strict validity.

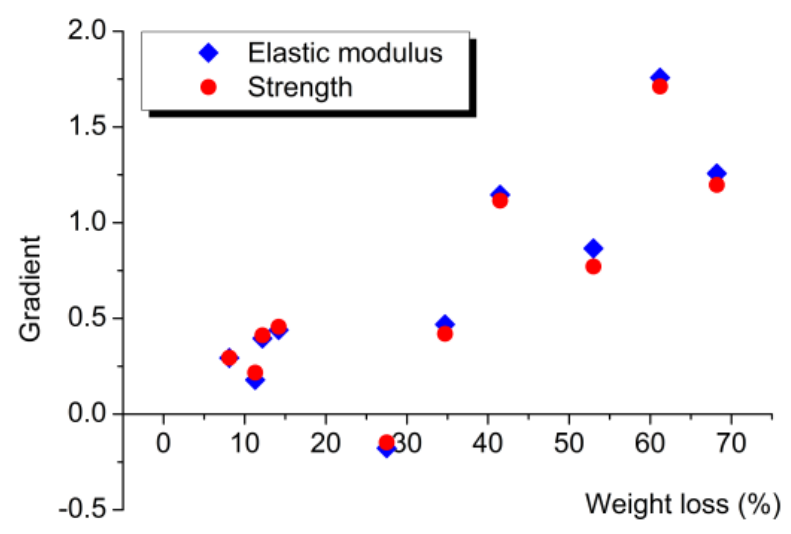

Figure 11 - The rate of change of strength and modulus with filler/matrix ratio at different weight losses of radiolytically oxidised HPB graphite. In the case for strength, the y-axis (labelled Gradient) presents the ratio of the strength change to the filler fraction change relative to the expected values of the strength and filler fraction at that weight loss. The modulus is presented similarly.

\section{Summary}

Due to the microstructural features of nuclear graphite, which has irregular shape pores with sizes at different length scales, a multi-scale model has been developed that uses direct input of the graphite microstructure. The model uses a novel concept for stress calculation in microstructures with many arbitrary shaped pores, based on the Cellular Automata technique that has advantages due to its flexibility and computational efficiency.

In support of this model, a simple approximation for the effects of porosity content and shape on the bulk elastic modulus has been presented, which can be applied to evaluate any arbitrary population of pores. A fracture algorithm, which uses the CA calculation for the stress concentration of pores and the effect of porosity on the stiffness of the porous material, has been developed. Using this model, fracture simulations have been performed for synthetic microstructures that comprise pores of spherical, ellipsoidal and arbitrary shapes. These demonstrate a significant effect of pore shape on strength, which is also observed in experimental studies in the literature, and supports the suitability of the proposed modelling technique.

In the simulation of the mechanical properties of radiolytically oxidised graphite with developing porosity, a small scale model provides the distributions of local elastic moduli and 
critical stresses, which are calculated for representative individual filler particles and matrix sub-volumes that have been characterised by high resolution X-ray computed tomography. A large scale model then operates at the length scale of the larger porosity $(>100 \mu \mathrm{m})$ with the given filler/matrix fractions for the corresponding weight loss value, taking into account the statistical distributions obtained by the small scale model. In addition to the microstructure input, only a pore-free value of Young's modulus and a critical parameter (stress or strain energy release rate) need to be used in the model for local fracture initiation. The resulting model describes well the effect of radiolytic oxidation on the degradation of elastic modulus and tensile strength. The model may be used to investigate the sensitivity of strength to variations in filler to matrix ratio, and could have applications to explain the response of different graphite grades to radiolytic oxidation.

\section{Conclusions}

The microstructures of the filler particles and matrix in irradiated oxidised Gilsocarbon graphite demonstrate different behaviour with increasing oxidation. The mean values of porosity within the filler particles are typically lower than in the matrix at the same weight loss, and microstructural changes in matrix are more extensive than in the filler. Consequently, the filler particles exhibit some stability to structure degradation in comparison with matrix with progressive radiolytic oxidation.

The developed multiscale model for fracture simulation in irradiated oxidised Gilsocarbon graphite shows a good agreement with available experimental data. The failure strength of the bulk graphite is dominated by the very significant reduction in the elastic modulus of the matrix, such that strain occurs predominantly in the continuous matrix that surrounds the filler particles; failure is thus predicted to occur in the matrix.

\section{Acknowledgements}

T.J.M. gratefully acknowledges the support of Oxford Martin School and EPSRC Grant EP/J019992/1), and Ye.V. acknowledges the support of EDF Energy Generation (GRA/GNSR 6061). Mr Alan Steer (EDF Energy Generation) is thanked for his helpful discussions. The authors also thank EDF Energy Generation for providing the data. Mr M. Jordan and Dr L Saucedo Mora are acknowledged for providing the large pore tomography data, obtained with the support of EDF Energy Generation (GRA/GNSR 6057) and EPSRC industrial CASE studentship 1237451. The views expressed in this paper are those of the authors, and not necessarily those of the project sponsors. 


\section{References}

[1] J.E. Brocklehurst, B.T. Kelly, Analysis of the dimensional changes and structural changes in polycrystalline graphite under fast neutron irradiation, Carbon 31(1) (1993) 155-178.

[2] P. Ramsay, Mechanical Property Measurements on AGR Core Graphite Using Electronic Speckle Pattern Interferometry in: G.B. Neighbour (Ed.) Modelling and Measuring Reactor Core Graphite Properties and Performance, RSC Publishing, Aston University, UK, 2013, pp. 61-68.

[3] J.E. Brocklehurst, R.G. Brown, K.E. Gilchrist, V.Y. Labaton, The Effect of Radiolytic Oxidation on the Physical Properties of Graphite, J Nucl Mat 35 (1970) 183-194.

[4] B.T. Kelly, T.D. Burchell, Structure-Related Property Changes in Polycrystalline Graphite under Neutron Irradiation, Carbon 32(3) (1994) 499-505.

[5] M.R. Bradford, A.G. Steer, A structurally-based model of irradiated graphite properties, Journal of Nuclear Materials 381(1-2) (2008) 137-144.

[6] S. Mrozowski, Mechanical strength, thermal expansion and structure of cokes and carbons, 1st and 2nd Conferences on Carbon, Waverly Press, 1956.

[7] K. Wen, J. Marrow, B. Marsden, Microcracks in nuclear graphite and highly oriented pyrolytic graphite (HOPG), J. Nucl. Mater. 381 (2008) 199-203

[8] N. Murdie, I.A.S. Edwards, H. Marsh, Changes in Porosity of Graphite Caused by Radiolytic Gasification by Carbon Dioxide, Carbon 24(3) (1986) 267-275.

[9] R. Coble, W.D. Kingery, Effect of Porosity on Physical Properties of Sintered Alumina, J Am Ceram Soc 39(11) (1956) 377-385.

[10] L.F. Nielsen, Strength and Stiffness of Porous Materials, J Am Ceram Soc 73(9) (1990) 2684-2689.

[11] M. Roessler, I. Odler, Investigations on the Relationship between Porosity, Structure and Strength of Hydrated Portland Cement Pastes. I. Effect of Porosity, Cement and Concrete Res 15 (1985) 320-330.

[12] R.M. Spriggs, A. Brissette, T. Vasilos, Effect of Porosity on Elastic and Shear Moduli of Polycrystalline Magnesium Oxide J Am Ceram Soc 45(8) (1962) 400.

[13] B.T. Kelly, Graphite - the most fascinating nuclear material, Carbon 20(1) (1982) 3-11.

[14] M.R. Joyce, T.J. Marrow, P. Mummery, B.J. Marsden, Observation of microstructure deformation and damage in nuclear graphite, Eng Fract Mech 75(12) (2008) 3633-3645.

[15] M. Mostafavi, T.J. Marrow, In situ observation of crack nuclei in poly-granular graphite under ring-on-ring equi-biaxial and flexural loading, Eng Fract Mech 78(8) (2011) 17561770 . 
[16] T.D. Burchell, A Microstructurally Based Fracture Model for Nuclear Graphite, Carbon 34(3) (1996) 297-316.

[17] M.R. Ayatollahi, J. Akbardoost, F. Berto, Size effects on mixed-mode fracture behavior of polygranular graphite, Carbon 103 (2016) 394-403.

[18] G.M. Laudone, C.M. Gribble, G.P. Matthews, Characterisation of the porous structure of Gilsocarbon graphite using pycnometry, cyclic porosimetry and void-network modeling, Carbon 73 (2014) 61-70.

[19] E.D. Eason, G.N. Hall, B.J. Marsden, G.B. Heys, Models of bending strength for Gilsocarbon graphites irradiated in inert and oxidising environments, J Nucl Mat 436(1-3) (2013) 208-216.

[20] E.D. Eason, G.N. Hall, B.J. Marsden, G.B. Heys, A model of Young's modulus for Gilsocarbon graphites irradiated in oxidising environments, J Nucl Mat 436(1-3) (2013) 201207.

[21] B.J. Marsden, G.N. Hall, 4.11 - Graphite in Gas-Cooled Reactors A2 - Konings, Rudy J.M, Comprehensive Nuclear Materials, Elsevier, Oxford, 2012, pp. 325-390.

[22] S.T. Kyaw, W. Sun, A.A. Becker, Effects of compositions of filler, binder and porosity on elastic and fracture properties of nuclear graphite, J Nucl Mat 457 (2015) 42-47.

[23] C. Berre, S.L. Fok, P.M. Mummery, J. Ali, B.J. Marsden, T.J. Marrow, G.B. Neighbour, Failure analysis of the effects of porosity in thermally oxidised nuclear graphite using finite element modelling, J Nucl Mat 381 (2008) 1-8.

[24] C. Berre, S.L. Fok, B.J. Marsden, L. Babout, A. Hodgkins, T.J. Marrow, P.M. Mummery, Numerical modelling of the effects of porosity changes on the mechanical properties of nuclear graphite, J Nucl Mat 352 (2006) 1-5.

[25] G.E. Smith, P. Flewitt, H.E.J.G. Schlangen, Multi-scale Modelling of Nuclear Reactor Core Graphite, Journal of Multiscale Modelling 05(01) (2013).

[26] B. Šavija, D. Liu, G. Smith, K.R. Hallam, E. Schlangen, P.E.J. Flewitt, Experimentally informed multi-scale modelling of mechanical properties of quasi-brittle nuclear graphite, Eng Fract Mech 153 (2016) 360-377.

[27] C.N. Morrison, M. Zhang, A.P. Jivkov, J.R. Yates, A Discrete Lattice Model of Quasibrittle Fracture in Porous Graphite, Materials Performance and Characterization 3(3) (2014) 414-428.

[28] C.N. Morrison, A.P. Jivkov, Y. Vertyagina, T.J. Marrow, Multi-scale modelling of nuclear graphite tensile strength using the site-bond lattice model, Carbon 100 (2016) 273282. 
[29] Lee Margetts, J.D.A. Mena, G.N. Hall, L.M. Lever, D.V. Griffiths, P.M. Mummery, Two tier parallel computation for stochastic thermo-mechanical modelling of nuclear graphite reactor cores, NAFEMS European Conference on Multiphysics Simulation, Manchester, 2014.

[30] M. Joyce, B. Davies, M.R. Bradford, Ongoing development of models to predict AGR core component condition, in: P.E.J. Flewitt, A.J. Wickham (Eds.) The 4th EDF Energy Nuclear Graphite Symposium, EMAS Publishing, Nottingham, 2015, pp. 195-212.

[31] Endorsement Statement for Compendium of CAGR Core and Sleeve Data and Methods. CSDMC/P28, (Data Sheet C1/2), 2003.

[32] Ye.Vertyagina, T.J. Marrow, Multifractal-based assessment of Gilsocarbon graphite microstructures, Carbon 109 (2016) 711-718.

[33] S.D. Brown, R.B. Biddulph, P.D. Wilcox, A Strength-Porosity Relation Involving Different Pore Geometry and Orientation, J Am Ceram Soc 47(7) (1964) 320-322.

[34] C.T. Herakovich, S.C. Baxter, Influence of pore geometry on the effective response of porous media, J Mater Sci 34 (1999) 1595-1609.

[35] L. Babout, B.J. Marsden, P.M. Mummery, T.J. Marrow, Three-dimensional characterization and thermal property modelling of thermally oxidized nuclear graphite, Acta Mater 56(16) (2008) 4242-4254.

[36] R.W. Rice, Evaluation and extension of physical property-porosity models based on minimum solid area, J Mater Sci 31(1) (1996) 102-118.

[37] L. Lei, J.L. Marin, M. Koslowski, Phase-field modeling of defect nucleation and propagation in domains with material inhomogeneities, Model Simul Mater Sc 21 (2013) 115.

[38] C. Fond, J. Kiefer, D. Mendels, J.B. Ferrer, H.H. Kausch, J.G. Hilborn, Influence of voids on the stress distribution and deformation behaviour of epoxies under uniaxial deformation, J Mater Sci 33 (1998) 3975-3984.

[39] B. Farida, S. Boualem, M.M.E. Amin, B. Benali, 3-D finite element analysis of stress concentration factor in spot-welded joints of steel: The effect of process-induced porosity, Comp Mater Sci 50 (2011) 1450-1459.

[40] E. Schlangen, Crack Development in Concrete, Part 2: Modelling of Fracture Process, Key Eng Mater 385-387 (2008) 73-76.

[41] D. Jauffrès, C.L. Martin, A. Lichtner, R.K. Bordia, Simulation of the elastic properties of porous ceramics with realistic microstructure, Model Simul Mater Sc 20(4) (2012) 1-18. 
[42] S.J. Zhou, W.A. Curtin, Failure of Fiber Composites: a Lattice Green Function Model, Acta Metall Mater 43(8) (1995) 3093-3104.

[43] S. Wolfram, A new kind of science, First Edition ed., Wolfram Media Inc, Champaign, 2002.

[44] V.K. Vanag, Study of spatially extended dynamical systems using probabilistic cellular automata, Phys Usp 42 (1999) 413-434.

[45] P.-Z. Pan, X.-T. Feng, J.A. Hudson, Study of failure and scale effects in rocks under uniaxial compression using 3D cellular automata, Int J Rock Mech Min 46 (2009) 674-685.

[46] D.D. Moiseenko, V.E. Panin, P.V. Maksimov, S.V. Panin, F. Berto, Material fragmentation as dissipative process of micro rotation sequence formation: Hybrid model of excitable cellular automata, International Conference on Physical Mesomechanics of Multilevel Systems, AIP Conference Proceedings, Tomsk, Russian Federation, 2014, pp. 427-430.

[47] A.Y. Smolin, N.V. Roman, I.S. Konovalenko, G.M. Eremina, S.P. Buyakova, S.G. Psakhie, 3D simulation of dependence of mechanical properties of porous ceramics on porosity, Eng Fract Mech 130 (2014) 53-64.

[48] S.G. Psakhie, D.D. Moiseyenko, A.Y. Smolin, E.V. Shilko, A.I. Dmitriev, S.Y. Korostelev, E.M. Tatarintsev, The features of fracture of heterogeneous materials and frame structures. Potentialities of MCA design, Comp Mater Sci 16(1-4) (1999) 333-343.

[49] L. Saucedo-Mora, T.J. Marrow, FEMME: A multi-scale Finite Element Microstructure MEshfree fracture model for quasi-brittle materials with complex microstructures, Eng Fract Mech 169 (2015).

[50] L. Saucedo-Mora, M. Mostafavi, D. Khoshkhou, C. Reinhard, R. Atwood, S. Zhao, B. Connolly, T.J. Marrow, Observation and simulation of indentation damage in a $\mathrm{SiC}-\mathrm{SiC}$ fibre ceramic matrix composite, Finite Elem Anal Des 110 (2016) 11-19.

[51] S. Timoshenko, G. Goodier, Theory of elasticity, McGraw-Hill Book Company, Inc., New York, Toronto, London, 1951.

[52] V.I. Astaf'ev, Y.N. Radaev, L.V. Stepanova, Nonlinear mechanics of destruction (in Russian), Samara University, Samara, Russian Federation, 2001.

[53] H.K. Lee, J.W. Ju, A Three-dimensional Stress Analysis of a Penny-shaped Crack Interacting with a Spherical Inclusion, International Journal of Damage Mechanics 16 (2007) 331-359.

[54] E.Z. Stowell, Stress and strain concentration at a circular hole in an infinite plate, NACA Technical note 2073 (1950) 1-14. 
[55] M.A. Sadowski, E. Sternberg, Stress concentration around a triaxial ellipsoidal cavity, J Appl Mech 16 (1949) 149-157.

[56] K.K. Phani, S.K. Niyogi, Young's modulus of porous brittle solids, J Mater Sci 22 (1987) 257-263.

[57] R.W. Rice, Comparison of stress concentration versus minimum solid area based mechanical property-porosity relations, J Mater Sci 28 (1993) 2187-2190.

[58] I.Y. Smolin, M.O. Eremin, P.V. Makarov, E.P. Evtushenko, S.N. Kulkov, S.P. Buyakova, Brittle Porous Material Mesovolume Structure Models and Simulation of their Mechanical Properties, International Conference on Physical Mesomechanics of Multilevel Systems, AIP Publishing LLC, 2014, pp. 595-598.

[59] A.P. Roberts, E.J. Garboczi, Elastic Properties of Model Porous Ceramics, J Am Ceram Soc 83(12) (2000) 3041-3048.

[60] C. Berre, S.L. Fok, B.J. Marsden, P.M. Mummery, T.J. Marrow, G.B. Neighbour, Microstructural modelling of nuclear graphite using multi-phase models, J Nucl Mat 380 (2008) 46-58.

[61] F.P. Knudsen, Effect of Porosity on Young's Modulus of Alumina, J Am Ceram Soc 45(2) (1964) 94-95.

[62] D.F. Porter, J.S. Reed, D.L. Iii, Elastic Moduli of Refractory Spinels, J Am Ceram Soc 60(7-8) (1977) 345-349.

[63] I.M. Pickup, B. McEnaney, R.G. Cooke, Fracture Processes in Graphite and the Effects of Oxidation, Carbon 24(5) (1986) 535-543.

[64] H. Imai, K. Fujii, T. Kurosawa, S. Nomura, Changes in Young's Modulus and Electrical Conductivity of Nuclear Grade Graphites Oxidized with Air, J Nucl Mat 118 (1983) 294-302. [65] Y. Vertyagina, T.J. Marrow, 3D Cellular Automata fracture model for porous graphite microstructures, Nucl. Eng. Des. (2016).

[66] D. Leguillon, R. Piat, Fracture of porous materials - Influence of the pore size, Eng Fract Mech 75(7) (2008) 1840-1853.

[67] D. Broek, Elementary engineering fracture mechanics, Forth Edition ed., Kluwer Academic Publishers, Dordrecht, 1986.

[68] E.S. Kalatur, S.P. Buyakova, S.N. Kulkov, I. Gotman, I. Kocserha, Porosity and Mechanical Properties of Zirconium Ceramics, J Silicate-Base Compos Mater 66(2) (2014) 31-34.

[69] J. Standring, B.W. Ashton, The Effect of Radiolytic Oxidation by Carbon Dioxide on the Porosity of Graphite, Carbon 3 (1965) 157-165. 
[70] Endorsement Statement for Compendium of CAGR Core and Sleeve Data and Methods. CSDMC/P28, (Data Sheet C9/1), 1994.

[71] H. Matsuo, Effect of Oxidation on Physical Properties of Neutron Irradiated Nuclear Grade Graphite, J Nucl Mat 138 (1986) 222-226.

[72] B.T. Kelly, P.A.V. Johnson, P. Schofield, J.E. Brocklehurst, M. Birch, U.K.A.E.A. northern division studies of the radiolytic oxidation of graphite in carbon dioxide, Carbon 21(4) (1983) 441-449.

[73] X. Aiping, S. Zhongyao, Direct Comparison between Tensile Strength and Flexural Strength of Ceramic/Metal Braizing Joint, Chin J Met Sci Technol 8 (1992) 30-34.

[74] A. Hodgkins, T.J. Marrow, M.R. Wootton, R. Moskovic, P.E.J. Flewitt, Fracture behaviour of radiolytically oxidised reactor core graphites: a view, Materials Science and Technology 26 (2010) 899-907.

[75] J. Brocklehurst, M. Darby, Concerning the fracture of graphite under different test conditions, Mater. Sci. Eng. 16 (1974) 91-106.

[76] N. Nemeth, A. Walker, E. Baker, P. Murthy, R. Bratton, Large-scale Weibull analysis of H-451 nuclear-grade graphite rupture strength, Carbon 58 (2013) 208-225. 